\title{
RATIO IN EARLY GREEK MATHEMATICS
}

BY D. H. FOWLER

\section{Contents}

1. Introduction

2. Arithmetike and logistike

3. The notion of ratio

4. Multiplication and addition of ratios

5. Pre-Eudoxan uses of incommensurable magnitudes and proportion theory

\section{Anthyphairesis}

7. Anthyphairesis and the discovery of incommensurability

8. Anthyphairetic ratio theory

9. Arithmetical implications of anthyphairesis

10. Theoretical and practical logistic

11. A new perspective on Book $X$

Appendix: Approximation by rational numbers, and continued fractions

\section{Notes}

Referencing. The following abbreviated titles are used for books to which frequent page-references are made:

Burkert, $L S=$ Lore and science in ancient Phythagoreanism.

Euclid, $E E=$ Elements, ed. and tr. T. L. Heath, 3 vols.

Heath, $H G M=A$ history of Greek mathematics, 2 vols.

Knorr, $E E E=$ The evolution of the Euclidean elements.

Thomas, $S G M=$ Selections illustrating the history of Greek mathematics, 2 vols.

van der Waerden, $S A=$ Science awakening.

Other references are by author and date. Full bibliographical details are listed at the end of the paper.

\section{Introduction.}

SOCRATES: Are there not two kinds of arithmetic, that of the people and that of philosophers?... And how about the arts of reckoning and measuring as they are used in building and in trade when compared with philosophical geometry and elaborate computations - shall we speak of each of these as one or two?

Protarchus: I should say that each of them was two. Plato, Philebus 56D-57E.

Received by the editors September 15, 1978.

1980 mathematics subject classification. Primary 01A20; Secondary 10A32, 10F20.

Key words and phrases. Greek mathematics, pre-Euclidean mathematics, Euclid's Elements, classification of incommensurable magnitudes, proportion theory, ratio theory, anthyphairesis, continued fractions, Euclidean algorithm. 
In this passage Plato distinguishes between practical arithmetic, the counting off of everyday objects - two oxen and two armies are examples he gives -and theoretical arithmetic, in which there is a single unit, identical in all its occurrences. This distinction is extended to measurement, contrasting practical measurements of areas with the highly developed Greek geometry; consider, for example, the theory of 'application of areas', the procedure for manipulating rectilinear plane figures. ${ }^{1}$ But what should we make of his further distinction between practical and theoretical calculations? Our interpretation of this will depend on and contribute to whatever reconstructions we propose for the kind of mathematics current in the Academy. I should like, in this article, to explore this kind of question in the setting of a drastic reinterpretation of pre-Euclidean mathematics recently proposed by W. R. Knorr. Knorr sees the construction and classification of incommensurable magnitudes (Books II and X of Euclid's Elements) as "a massive project which engaged the best efforts of the most notable fourth-century mathematicians: Theodorus, Theaetetus, Archytas and Eudoxus,"2 and he extends the proposal by Becker and others ${ }^{3}$ that, before the development of Book V-type proportion theory by Eudoxus, proportion was characterised using the 'Euclidean' subtraction algorithm, in a procedure called anthyphairesis. But we make one modification to Knorr's account: we shall argue that this anthyphairetic definition, introduced by Theaetetus and used by Eudoxus before his discovery of the more powerful and general Book V Definition 5, was used to develop a theory of ratio, not of proportion, and we examine this conjecture against the available evidence.

For reasons that we set out (in \$10), the anthyphairetic theory, once superceded, would be forgotten and misunderstood; therefore contemporary testimony is the only reliable guide. Thus, from the following approximate limiting dates ${ }^{4}$ : Plato (428-347); Theaetetus (414-369); Eudoxus (395-340); Aristotle (384-322); and the discovery of Book V-type proportion theory ${ }^{5}$ around 350 , we see that the writings of Plato and his associates in the Academy, where in fact the developments were taking place, provide the best evidence. Relevant passages are considered in $\$ \S 2,5,8$, and 10 . Secondly, since the bulk of our knowledge of fourth-century mathematics comes to us via Euclid's Elements (c.300), we examine that for vestiges of the anthyphairetic theory in $\$ \S 3,4,6$, and 11 , and point out the different characteristics of ratio and proportion theory ( $\$ 33$ and 10$)$. All this discussion rests on an appreciation of the historical role of anthyphairesis and an understanding of its mathematical implications. The procedure itself, as found in the Elements, is described in \$6; two basic geometrical calculations using it are performed in $\$ 7$; and the historical evidence for it is set out in $\S 8$;

1 For a description of the application of areas, and texts, see Heath, HGM I, pp. 150-153; Thomas, $S G M$ I, pp. 186-215; and van der Waerden, $S A$, pp. 118-124.

2 Knorr, $E E E$, p. 1.

3 Also Zeuthen and Dijksterhuis; see Knorr, $E E E$, pp. 255-261 (references are given in n. 19) and van der Waerden, $S A$, pp. 175-179.

4 For references to the question of dating, see Knorr, EEE, p. 297 n. 93.

5 For the dating of this discovery, see Knorr, EEE, pp. 94-95 and pp. 284-285. Our argument will not be altered in any significant way by variations in these dates. 
$\$ 9$ shows how the apparently elaborate arithmetic that anthyphairesis demands can be performed within the range of techniques available to fourthcentury Greek mathematics, and how certain important features can readily be observed; $\$ 10$ introduces a new kind of 'literary' evidence for the role of anthyphairesis, and $\$ 11$ describes how it gives a new motivation for the classification of irrationals given in Book X of the Elements. Finally, the mathematical theory behind anthyphairesis is developed in the Appendix using a series of equivalent algorithms.

It will be superfluous for me to point out the extent to which my inspiration, elucidation, and raw material derive from the studies of Wilbur Knorr. I should like to thank him for the interest and information he has extended to me during the past years, and his recent enthusiasm over a much more modest proposal which has grown into the programme outlined here. The second person who has influenced, encouraged, and shared his knowledge and enthusiasm with me is Malcolm Brown. Without these two, I should have got nowhere.

Many other people have helped in different ways during the gestation of these ideas: colleagues and students, family and friends. The final step of writing a first draft was made possible by a visit to the Institute des Hautes Études Scientifique, Bures-sur-Yvette. Detailed comments were made by Malcolm Brown, Jim Franklin, Wilbur Knorr, Barry Mazur, Ian Mueller, and Tom Whiteside, and the final result benefitted greatly from their sceptical attention. I would like to thank all these people for their help, interest, and encouragement.

2. Arithmetike and logistike. Within the surviving Greek classical mathematical tradition, number (arithmos) always denotes a positive integer, ${ }^{6}$ of ten excluding unity, and arithmetic (arithmetike) corresponds to our theory of numbers, though these words have a more concrete sense than our use of 'number', being more connected with the actual process of counting and implying the existence of things, possibly idealised units, being counted, as with the English words 'couple', 'trio', 'dozen', etc. ${ }^{7}$ The use of the ratio $\left(\log o s^{8}\right)$ of two numbers does appear in the more informal proofs of Archimedes but there are no surviving attempts to incorporate the manipulation of fractions within a formal treatment. In Euclid's Elements, for example, there is very occasional use of 'halves' or 'thirds', as in I, 47, and XII, 10, and later interpolated elaborations of the Common Notions, but the explicit operations of addition and multiplication of the ratios of two numbers are never considered. ${ }^{9}$

6 We shall adhere to this terminology throughout the main part of the paper. In the Appendix, number will be used in the sense of 'real number'.

7 For a full discussion of arithmos, see J. Klein (1968), passim.

8 See von Fritz (1945), pp. 250 and $260-263$ for a valuable discussion of the word logos and associated words in a mathematical context.

9 Pace the opinion of van der Waerden, $S A$, p. 116, that "Theoretical logistic deals especially with the study of numbers in their mutual ratios, exactly the sort of thing treated in Book VII ... "; Book VII is mainly Plato's theoretical arithmetic. See §4. 
Plato makes frequent reference to mathematics, ${ }^{10}$ singling out for special mention the subject of 'calculation' (logistike and logismos; hereafter we shall transcribe these as 'logistic') as a clearly defined and important intellectual discipline; sometimes the context gives a contrast with practical calculation indicating that a theoretical study corresponding to the practical manipulation of fractions is being considered-the passage from Philebus with which we started, is such an example. An important passage in the Republic (524D $-526 \mathrm{C}$ ), where he discusses the proper education for the guardians of the state, illustrates these points. Here he assigns arithmetic and logistic, with a strong emphasis on logistic, to first place in the curriculum:

It is befitting, then, Glaucon, that this branch of learning [logistike] should be prescribed by our law and that we should induce those who are to share the highest functions of state to enter on that study of calculation and take hold of it, not as amateurs, but to follow it up until they attain to the contemplation of the nature of number, by pure thought, not for the purpose of buying and selling.... . And it occurs to me, now that the study of reckoning (logistike) has been mentioned, that there is something fine in it, and that it is useful for our purpose in many ways, provided it is pursued for the sake of knowledge and not for huckstering ... that it strongly directs the soul upwards and compels it to discourse about pure numbers, never acquiescing if anybody proffers to it in the discussion numbers attached to visible and tangible bodies.

This passage then continues with what appears to be a clear indication that fractional calculations are not reduced to integer arithmetic by dividing up the unit:

For you are doubtless aware that experts in this study, if anyone attempts to cut up the 'one' in argument, laugh at him and refuse to allow it; but if you mince it up, they multiply, always on guard lest the one should appear to be not one but a multiplicity of parts.

Finally the difficulty of the subject is emphasized:

And, further, as I believe, studies that demand more toil in the learning and practice than this we shall not discover easily nor find many of them.

Here are some other instances: Hippias is described as skillful at arithmetic, logistic, geometry, and astronomy (Lesser Hippias 366C-368A), and similarly Theodorus is an expert in geometry, astronomy, logistic, music, and all the liberal arts (Theaetetus 145A-B); and geometry, astronomy, and logistic

10 For a general summary of the role of mathematics in ancient Greece, with references, see Burkert, LS, Chapter VI, 1, esp. pp. 420-427. For Plato, see Knorr, EEE, pp. $87-96$. 
are compared with hunting as arts of the pursuit and capture of real meaning (Euthydemus 290B-C). Elsewhere Plato gives comparisons between the subject-matter of arithmetic and logistic; we shall return to these later. ${ }^{11}$

A surviving fragment of Archytas ${ }^{12}$ corroborates this interest in logistic as a theoretical discipline, related to geometry:

Logistic seems to be far ahead of the other arts in relation to wisdom or philosophy, nay it seems to make the things of which it chooses to treat even clearer than geometry does; moreover it often succeeds even where geometry fails.

Another instance comes from Aristotle, ${ }^{13}$ quoted by Iamblichus:

Those who concern themselves with geometry and logistic and the other sciences have from small beginnings made by now such progress in a very short time as no other field has made in any of the arts.

There has been considerable discussion of the roles of arithmetic and logistic within Greek intellectual activity. The later sense of logistic is quite clearly that of practical numerical calculations, and many commentators, both ancient and modern, treat this as its only meaning. For example, Proclus writes: ${ }^{14}$

But others, like Geminus, ${ }^{15}$ think that mathematics should be divided differently; they think of one part as concerned with intelligibles only and of another as working with perceptibles and in contact with them .... O Of the mathematics that deals with intelligibles they posit arithmetic and geometry as the two primary and most authentic parts, while the mathematics that attends to sensibles contains six sciences: mechanics, astronomy, optics, geodesy, canonics, and calculation ... . Geodesy and calculations are analogous to these sciences [i.e. geometry and arithmetic], since they discourse not about intelligible but about sensible numbers and figures ... . Nor does the student of calculation consider the properties of number as such, but of numbers as present in sensible objects ....

A similar classification, due to Anatolius, is given by Heron in his Definitions. $^{16}$ The inadequacy of this interpretation for the subject to which Plato, Archytas, and Aristotle are referring is obvious: their study is theoretical

$11 \quad$ See $\$ 10$.

12 Fragment B4, Diels and Kranz (1951-1952) I, p. 438. Burkert, $L S$, p. 220 n. 14 regards this fragment as spurious since "the assertion that logistic helps where geometry fails is nonsensical mathematically". By providing a clear meaning for the phrase, we shall remove this objection.

13 Fragment 53, quoted in Burkert, $L S$, p. 423.

14 Proclus' Commentary pp. 38-39 (Friedlein's pagination).

15 Geminus' date and birthplace are uncertain; he was probably a first-century Stoic philosopher. See Heath, HGM II, pp. 222-223.

16 Extracted in Thomas, $S G M I$ I, pp. 18-19. 
(corresponding to Proclus' "concern with intelligibles only"), and must refer to a very special kind of calculation, if calculation it be. ${ }^{17}$

Our proposals may also help to explain the method by which fractions were treated. During the early classical period the Greeks handled fractions as did the Egyptians, by expressing proper fractions (with the exception of $\frac{2}{3}$ ) as sums of submultiples (e.g. $\frac{3}{4}=\frac{1}{2}+\frac{1}{4}$ ). While there does not appear to have been any early theoretical understanding of the other operations on fractions, reciprocation (describing by prefixing hypo) and the epimoric fractions $(m n+$ $m) / m n$ do seem to have been familiar. These will behave simply with respect to an algorithm to be described in $\$ 9 .^{18}$

3. The notion of ratio. It is a curious, obvious, and unexplained fact that the Elements does not contain a precise definition of ratio, though the word logos is used frequently with this meaning. Book V, Definition 3 introduces it:

A ratio (logos) is a sort of relation in respect of size between two magnitudes of the same kind,

but its sense is defined in the celebrated Definition 5 where what is actually considered is the equality of two ratios. Definition 6 then introduces retrospectively an alternative terminology:

Let [four] magnitudes which have the same ratio be called proportional (analogon),

and Book $\mathrm{V}$ goes on to study proportionality among magnitudes; so proportonality is a relationship that may or may not hold or be relevant.

To emphasise this difference between ratio and proportion: given four objects $a, b, c$, and $d$, we can always answer either 'Yes, they are in proportion'; or 'No, they are not in proportion'; or 'The idea of proportion is irrelevant (since $a$ and $b$, or $c$ and $d$, cannot be compared)'. The procedure of Book $\mathrm{V}$ does not assign meanings to $a: b$ and $c: d$ separately and then assert that they are equal-for this reason, we shall use the abbreviation $a: b:: c: d$ for proportions, rather than $a: b=c: d$. A ratio is an independent meaning for $a: b$ and, in most treatments, it follows a definition of proportion and corresponds to an 'equivalence class' of proportions; this step is in no sense considered in the Elements, no alternative definition of ratio apart from $\mathrm{V}$, Definition 3 is proposed, and so ratios can only be meaningfully considered there within a proportion. ${ }^{19}$

17 For details of Greek methods of calculation, see Heath, HGM I, pp. 26-64, and Thomas, $S G M$ I, pp. $28-63$. We shall not deal with this question here.

18 For the suggestion that the epimoric terminology derives from the calculation of interest, see Burkert, $L S$, pp. 438-440. But the problem still remains: apparently only this restricted set of ratios of numbers was well understood.

19 Heath, in his Introductory note to Book VI (Euclid, EE II, p. 187) writes "This limitation [on geometrical algebra] disappears as soon as we can represent any general quantity, corresponding to what we denote by a letter in algebra, by a ratio; and this we can do because, on the general theory of proportion established in Book V, a ratio may be a ratio of two incommensurable quantities as well as commensurables. Ratios can be compounded [i.e. multiplied; see the next section] ad infinutum, and the division of one ratio by another is equally easy, since it is the same thing as compounding the first ratio with the inverse of the second." This passage is sustained nonsense because, to repeat, the idea of a ratio is never defined in the Elements. We shall see later that, even with our reconstructed definition of ratio, they cannot easily be compounded. 
Proportionality among four numbers is defined, independently and differently, in VII, Definition 20:

Numbers are proportional when the first is the same multiple, or the same part, or the same parts, of the second that the third is of the fourth. ${ }^{20}$

Commentators rarely point out the unsatisfactory nature of this definition: it is a vivid, though incomplete, description of four numbers in proportion, and not the mathematical criterion needed for the foundation of a theory. (Compare it with V, Definition 5. This latter, as a description, is almost impenetrable, though its latent power and scope are enormous.) But, even as a description, it is flawed since the terms in which it is couched-multiple, part, or parts-are themselves defined in terms of a basic undefined operation of 'measuring', in VII, Definitions 3, 4, and 5, parallel definitions to V, Definitions 1 and 2. ${ }^{21}$ Again we find that although ratio is not mentioned within the arithmetical definitions of Book VII, the word appears frequently throughout the arithmetical Books VII to IX.

A third terminology, not formally defined but also used throughout the Elements, is based on the phrase translated as 'have (or are) to one another'. A good example is VI, 1:

Triangles and parallelograms which are under the same height are to one another as their bases.

When the different definitions of proportion relating to magnitudes and numbers are reconciled in $\mathrm{X}, 5$ :

Commensurable magnitudes have to one another the ratio which a number has to a number,

neither the enunciation, nor the proof (which, as is well known, is deficient in its use of the two definitions ${ }^{22}$ ) uses the terminology of proportionality associated with the formal definitions. ${ }^{23}$

In $\$ 8$ we shall put forward the proposal that a different, explicit definition of ratio was in use before the development by Eudoxus of a general theory of proportion. The widespread use by Euclid of the terminology of ratios, and the inconsistancies in proofs that result from this, then appear as relics of this superceeded procedure.

4. Multiplication and addition of ratios. Operations corresponding to the multiplication of ratios do occur within the Elements, with treatments that are worthy of note. First observe that although the idea of taking the product of two ratios is never defined-nor could it be since ratio itself is not defined, but only the equality of two ratios-the operation is used in VI, 23:

20 For example, 6 is a multiple of 2,2 is a part of 6,4 is parts of 6 . Note how this omits the case of 6 and 4.

21 See also $\$ 6$.

22 See Knorr, EEE, pp. 253-255.

23 Ratios are also 'defined' and manipulated within Euclid's Data and On divisions. See Heath, HGM I, pp. 421-430 and Thomas, SGM I, pp. 478-479. 
Equiangular parallelograms have to one another the ratio compounded of the ratio of their sides.

The terminology is defined in an interpolated corrupt Definition 5:

A ratio is said to be compounded of ratios when the size of the ratios are multiplied into themselves ${ }^{24}$

but this is scarcely explicit.

There could not be a general proposition in Book $\mathrm{V}$ on the compound of two proportions between magnitudes, since the result might seem to involve the product of the magnitudes, which would not in general be defined. Particular cases do however occur; significantly ${ }^{25}$, the very first proposition in Book $\mathrm{V}$ to deal with proportions, Proposition 4, proves that (paraphrasing) if $a: b:: c: d$, then $m a: n b:: m c: n d$, describing the compound of a ratio of numbers and a proportion between magnitudes. Another case occurs within the combined definition and proposition, V, Definition 17:
A ratio ex aequali arises when, there being several magni- tudes and another set equal to them in multitude which taken two and two are in the same proportion, as the first is to the last among the first magnitudes, so is the first to the last among the second magnitudes;
Or, in other words, it means taking the extreme terms by virtue of the removal of the intermediate terms.

This result, that if $a: b:: a^{\prime}: b^{\prime}, b: c: b^{\prime}: c^{\prime}, \ldots, d: e:: d^{\prime}: e^{\prime}$, then $a: e:: a^{\prime}: e^{\prime}$, is proved as V, 22, and is used in the proof of VI, 23, cited above, where line segments are constructed representing the given ratio, so contrived that the procedure ex aequali can be applied. A special case of VI, 23 occurs as VI, 14 and deals with equiangular parallelograms having the same area; the operation considered here is reciprocation (VI, Definition 2) and, although this definition is also corrupt, a satisfactory version of VI, 14 does not pose the same problem of meaning as occurred with VI, $23 .{ }^{26}$

Multiplication and addition of ratios of numbers would apparently fall within the scope of Book VII; indeed closely related results do appear, but in a curiously convoluted form. For example, Proposition 10 states:

If a number be parts of a number, and another be the same parts of another, alternately also, whatever parts or part the first is of the third, the same parts or the same part will the second also be of the fourth.

24 Heath's translation: "A ratio is said to be compounded of ratios when the sizes of the ratios multiplied together make some (?ratio, or size)" (Euclid, EE II, pp. 189-190). See also Heath, HGM I, p. 393: "Euclid has never defined 'compound ratio' or the 'compounding of ratios'; but the meaning of the terms and the way to compound ratios [of magnitudes?] are made clear in this proposition."

Compounding ratios is not to be confused with the composition of ratios, V, Definition 14; see that note in Euclid, EE II, pp. 134-135. 
This result, paraphrased, states that $(m / n) a:(m / n) b:: a: b$; the case $m=1$ is treated separately as Proposition 9, with an almost identical enunciation. Subsequently, Proposition 17 deals with the case $n=1$, but with such a different enunciation that a different operation is closely being considered:

If a number by multiplying two numbers make certain numbers, the numbers so produced will have the same ratio as the numbers multiplied.

At no point in the Elements, or in the surviving corpus of classical Greek mathematics does anything remotely related to a formal treatment of addition of ratios of numbers occur.

Why then do we not find, within Euclid or elsewhere, a treatment of logistic dealing with the basic operations on ratios? Such a question is not out of place, since we do find Euclid attempting to introduce some of these manipulations. We shall give a reason in $\$ 9$, where we shall see that what would be required for such a book would lie far outside the scope of Greek mathematics.

5. Pre-Eudoxan uses of incommensurable magnitudes and proportion theory. There is no evidence of restraint on the part of mathematicians, or concern among philosophers over the use of incommensurable ratios in the preEudoxan period. ${ }^{27}$ For example, while the fact of incommensurability is explicitly dealt with in Plato's Theaetetus (147C-148B), the only place where distress over ignorance or error is expressed is in the Laws (819D-820D), a late dialogue written during the time of Eudoxus. ${ }^{28}$ Plato here must surely be reacting to new discoveries within an existing theory of incommensurability, not expressing his ignorance of the fact or immediate consequences of the original discovery of the phenomenon.

As an example of mathematical developments we have, for instance, the impressive classification of quadratic irrationals in Book X of the Elements. Pappus $^{29}$ describes this as follows:

The aim of Book X of Euclid's treatise on the Elements is to investigate the commensurable and the incommensurable, the rational and the irrational continuous quantities. This science had its origin in the sect of Pythagoras, but underwent an important development at the hands of the Athenian Theaetetus, who had a natural aptitude for this and for other branches of mathematics most worthy of admiration. One of the most happily endowed of men, he patiently pursued the investigation of the truth contained in these [branches of] science, as Plato bears witness for him in the book which he called after him, and was in my opinion the chief means of

27 For discussion of the lack of reliable evidence for a 'foundations-crisis' in pre-Eudoxan mathematics, see Burkert, $L S$, pp. 455-465, and Knorr, $E E E$, pp. 306-312.

28 For a discussion of this passage, see Knorr, $E E E$, p. 22, esp. n. 11, 94-95, 102 n. 62, and 285.

29 This commentary survives only in Arabic; see the edition and translation by Junge and Thomson (1930), Pt. I, par. 1. 
establishing exact distinctions and irrefragable proofs with respect to the above mentioned quantities. For although later the great Apollonius whose genius for mathematics was of the highest possible order, added some remarkable species of these after much laborious application, it was nevertheless Theaetetus who distinguished the powers which are commensurable in length from those which are incommensurable ... as is stated by Eudemus the Peripatetic.

Although Pappus may be a biased commentator, writing 700 years after the event, his reference to Eudemus ${ }^{30}$ gives validity to this account. At the very least, therefore, this passage indicates that Eudemus, as Pappus' source, saw no difficulty in results about incommensurable magnitudes being established at a time before Book $\mathrm{V}$ proportion theory.

Another important passage is the long fragment of Eudemus describing Hippocrates' quadrature of lunes quoted by Simplicius. ${ }^{31}$ We find that

He made his starting point, and set out as the first of the theorems useful to his purpose, that similar segments of circles have the same ratio as the squares on their bases. And this he proved by showing that the squares on the diameters have the same ratios as the circles.

The text in Simplicius continues by explaining that ratio here is defined as for commensurable magnitudes, though he expresses it only in terms of 'part of'; several attempts have been made to extract Eudemus' original text from the quotation, and some argue that this subsequent passage is a later interpolation. If this is the case, the quoted passage indicates again how Eudemus had no hesitation in attributing results on incommensurable ratios to an earlier period.

Further evidence comes from the work of Archytas. In his geometrical construction for the duplication of the cube ${ }^{32}$ we get an explicit manipulation of similar triangles, and hence a proportion in which the terms are incommensurable. Then, in his work on music theory, the nonexistence of certain ratios of integers implies the existence of incommensurable line segments.

6. Anthyphairesis. The 'Euclidean' subtraction algorithm - since the proce-

30 Eudemus of Rhodes, Aristotle's pupil, wrote a History of Mathematics that became a standard reference throughout ancient times. Although this book, and its companion History of Astronomy, is now lost, many passages are quoted by other authors, and such passages, once identified, are considered to be our main reliable source of information about pre-Euclidean mathematics.

31 Extracted in Thomas, SGM I, pp. 234-253. The quotation starts "I shall set out what Eudemus wrote word for word, adding only for the sake of clearness a few things taken from Euclid's Elements on the account of the summary style of Eudemus, who set out his proofs in abridged form in conformity with the ancient practice." The problem of identifying the original text is discussed in Heath, HGM I, pp. 183-191.

32 Extracted in Thomas, SGM I, pp. 284-289. Again the source, Eutocius, refers it to Eudemus. 
dure clearly antedates Euclid, a more neutral terminology is preferable ${ }^{33}$ -appears applied to numbers in VII, 1, 2, and 3; while in X, 2, 3, and 4 it is used on magnitudes. Here, for instance, is X, 2:

If, when the less of two unequal magnitudes is continually subtracted in turn from the greater, that which is left never measures the one before it, the magnitudes will be incommensurable.

The procedure being described in the phrase is continually subtracted in turn from', anthyphairesis, ${ }^{34}$ is as follows: Given two homogeneous magnitudes $A$ and $B$ (think of $A$ and $B$ as line segments) with $B$ smaller than $A$, suppose $B$ goes into $A$ some number $n_{0}$ times leaving a remainder $A_{1}$ less than $B$; now repeat the procedure with $B$ and $A_{1}$, giving rise to a second number $n_{1}$ and magnitude $B_{1}$. If, at some stage, the current smaller magnitude goes precisely into the larger magnitude - 'measures it' in Euclidean terminology, undefined $^{35}$ and used with the same force as our 'goes into'-then this current smaller magnitude measures the magnitude before, which then measures the magnitude before it, and so on, and so it measures both original magnitudes, which are therefore commensurable (X, Definition 1$)$ and the process terminates; this is $\mathrm{X}, 3$. Conversely if the magnitudes are commensurable, so have a common measure $C$, the process will terminate $(X, 2)$. This follows because we can see that any common measure of $A$ and $B$ must also measure the remainder $A_{1}$; hence the first step replaces $A$ and $B$ by a smaller pair $B$ and $A_{1}$, which also have common measure $C$. Repeating this process sufficiently many times will decrease the magnitudes until, it can be shown ${ }^{36}$ using $\mathrm{X}, 1$, they become less than $C$, at which a contradiction is manifest.

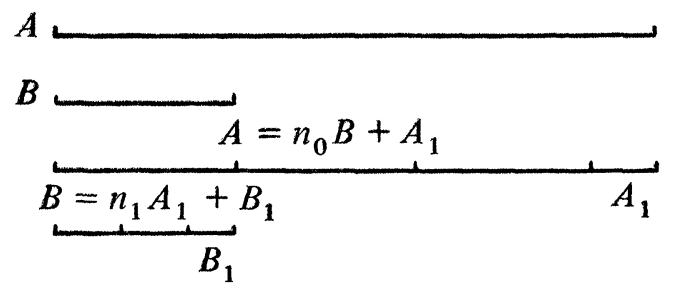

etc.

\section{Figure 1}

Similar results hold when the procedure is applied to two numbers, where the commensurable/incommensurable distinction is replaced by the existence or not of a common factor. Since, at each step, the numbers decrease, we see

33 In view of Pappus' testimony, the 'Theaetetan subtraction algorithm' would be a better name. That Euclid's role was to downgrade the importance of the algorithm, as we shall argue in $\$ 11$, adds irony to the misattribution.

34 We use the word in a substantive form ("the anthyphairesis of two magnitudes"); it occurs in Euclid only in a verbal form (e.g." ... when the less of two unequal magnitudes is continually subtracted in turn from the greater ..." ").

35 Introduced in V, Definition 1: "A magnitude is a part of a magnitude, the less of the greater, when it measures the greater".

36 An extended study by Knorr of bisection arguments is in progress. 
immediately in this case that the procedure will always terminate, and the remainder at the penultimate step will be the highest common factor.

We call this procedure 'anthyphairesis', and refer to the sequence $n_{0}, n_{1}, \ldots$ as 'the anthyphairesis of $A$ and $B$ ', sometimes writing $\operatorname{Anth}(A, B)$ $=\left[n_{0}, n_{1}, n_{2}, \ldots\right]$.

7. Anthyphairesis and the discovery of incommensurability. It has been suggested that anthyphairesis provided the context for the original discovery of incommensurable magnitudes, particularly since the procedure is easily performed on a diagonal and side of the pentagon and leads to the inscribed pentagram, a figure closely associated with the Pythagoreans. ${ }^{37}$ The pentagram is based on an isosceles triangle whose base angles are double the remaining angle. The construction of such a triangle ( $A B C$ and $A E G$ are typical ones in Figure 2) occurs as IV, 10, and is based on II, 11, the line cut in extreme and mean ratio or the 'golden section'. Consequences are that any two intersecting diagonals of a pentagram cut each other in extreme and mean ratio; all the angles in Figure 2 are multiples of the basic angle $B A C$, $36^{\circ}$; and many triangles are isosceles. (Another approach, exploiting the symmetries of the figure, uses the many parallelograms to be found within the

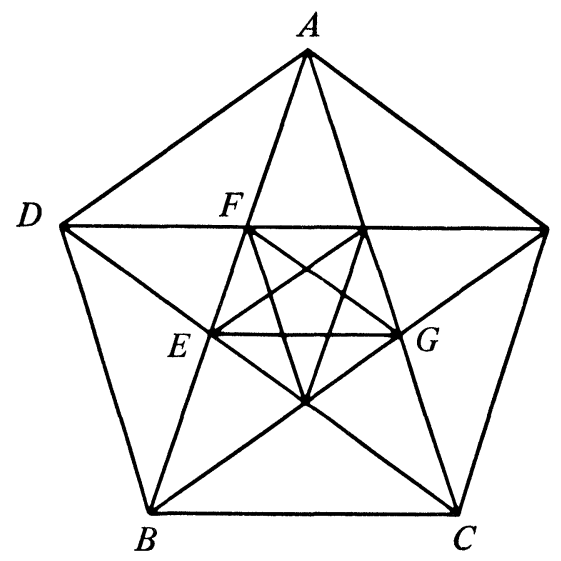

Figure 2

figure.) Hence performing anthyphairesis on the diagonal and side of a pentagon (say $A B$ and $A D$ ) is the same as anthyphairesis of the mean and extreme ratio, and it is immediately read off from the diagram:

$$
\begin{array}{r}
A B=A E+E B=A D+E B \text { where } E B=A F \text { is less than } A D . \\
A D=A E=A F+F E=F G+F E \text { where } F E, \text { a side of the } \\
\text { inner pentagon, is less than } F G, \text { a diagonal. }
\end{array}
$$

Thus the anthyphairesis of the larger pentagon leads, after two steps, to the anthyphairesis of the inner pentagon, and gives the sequence one, one, one, etc. $^{38}$ Therefore the anthyphairesis continues indefinitely and, using this as

37 See, for example, von Fritz (1945); but this is strongly disputed by Knorr. See Knorr, $E E E$, pp. 29-36 and 118-126, and Burkert, $L S$, p. 459.

38 Note that although the sequence does not terminate, it has been calculated in a finite number of steps, all ruler and compass constructible. 
the criterion of incommensurability, the diagonal and side are incommensurable. Or one can argue by reductio: if they were commensurable, their ratio could be expressed as a ratio of integers. But this process applied to integers terminates, and this is a contradiction.

The procedure can also be applied to a square where it gives results closely related to the 'side and diameter numbers', described by Proclus and Theon of Smyrna. Proclus says

The Pythagoreans proposed this elegant theorem about the diameters and sides, that when the diameter receives the side of which it is diameter it becomes a side, while the side, added to itself and receiving its diameter, becomes a diameter. ${ }^{39}$

He then goes on to refer it to Euclid II, 10, thus placing it in a geometrical context. The passage might be a vivid description of the modified Meno-like figure $^{40}$ shown (Figure 3). From this we can read off the anthyphairesis:

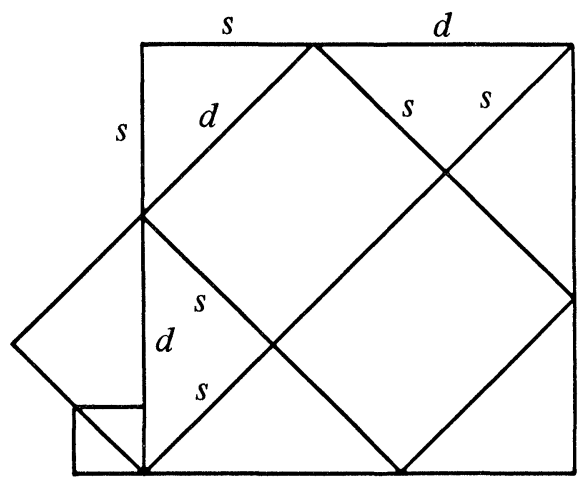

Figure 3

diameter of largest square $=$ side of largest square

+ side of smaller oblique square;

side of largest square $=$ twice side of smaller square

+ (diameter minus side of smaller square)

and, by scaling down the figure to apply now to the smaller square, we see that the last remainder is the side of the smallest square in the corner. Hence the procedure repeats, giving as anthyphairesis a sequence one, two, two, two, etc. ${ }^{41}$

39 See Thomas, $S G M$ I, pp. $132-139$ for the full texts. The side and diameter numbers are defined by $s_{n+1}=s_{n}+d_{n}, d_{n+1}=2 s_{n}+d_{n}$, with $s_{0}=d_{0}=1$.

40 See the Meno $(82 \mathrm{~A}-85 \mathrm{C})$ where Socrates persuades an untutored slave to double a square. This type of reconstruction is discussed by Burkert, $L S$, p. 430; Heath, in Euclid, $E E$ I, pp. 400-401; Knorr, $E E E$, pp. 31-36; and van der Waerden, $S A$, p. 127, none of whom point out the similarity to the Meno figures, the only diagrams occurring in the Dialogues. All geometrical reconstructions relating to the side and diameter numbers are speculative; but if, as Knorr argues (EEE, p. 174-180) the 'Chinese' proof of Pythagoras' Theorem-another variant on the Meno figure-should be interpolated between II, 8 and 9 then this indicates a coherent collection of results on squares or rectangle situated obliquely within a square.

41 Again finitely ruler and compass constructible. 
This is not the place to discuss the relative merits of different proposals for a possible context of the first discovery of incommensurability. ${ }^{42}$ Let me only say, briefly, that there are serious objections to an anthyphairetic discovery: both the possibility of a nonterminating anthyphairesis, and the subsequent implications of this require a greater understanding than arguments based on, for example, Plato's Meno figure and relating to the side and diagonal of a square. (We shall, however, go on to argue that anthyphairesis did play an important part in the fourth-century studies of incommensurability, and in the arithmetical techniques of defining, manipulating, and approximating ratios of numbers.) Also many scholars now argue that the well-known proof, referred to by Aristotle ${ }^{43}$ in terms that if the side and diameter are commensurable, then one may show that odd numbers equal even numbers, was not how it was originally discovered. Whatever actually happened, it makes no difference to the thesis argued here.

8. Anthyphairetic ratio theory. We now consider the suggestion that the ratio of two numbers or magnitudes was defined by their anthyphairesis, ${ }^{44}$ or some procedure intimately connected with anthyphairesis, and two ratios are equal if they have the same anthyphaireses. For example, we have shown that the anthyphairesis of the diameter and side of a pentagon is a sequence of ones, and of a square the sequence: one, two, two, two, ... .

For ratios of numbers and of commensurable magnitudes, the process gives rise to a finite sequence of terms of which only the first may be zero; in fact anthyphairesis is precisely the procedure used to determine their common measure (VII, 2 for numbers, X, 3 for magnitudes). Each step of the operation is straight-edge and compass constructible when applied to lines and rectilinear areas, using the theory of application of lines ${ }^{45}$ and areas, whilst constructible extensions to further kinds of magnitudes such as circles or polyhedral solids would have been consequences of solutions of the classical Greek problems of squaring the circle or doubling the cube. Consideration of the nonterminating anthyphairesis of incommensurable magnitudes would lead to serious philosophical problems $\mathrm{s}^{46}$ and technical mathematical difficulties, but we have shown how some important ratios are determined in a finite number of constructible steps, and there would be no difficulty in handling the procedure at an informal level, as we shall demonstrate.

Let us examine the evidence that such a definition was used:

First, a passage from Aristotle's Topics (158b29):

It would appear that in mathematics too some things are difficult to prove owing to the want of a definition, for instance that the line parallel to the side and cutting the plane figure divides similarly the base and the area. But once

42 See Knorr, $E E E$, pp. 21-61 for an account of the discovery, with references, and especially pp. 29-36 for a discussion of anthyphairesis.

43 Prior Analytics I. 23, 41a29, extracted in Thomas, SGM I, pp. 110-111.

44 See Knorr, $E E E$, pp. 255-273 for a full account. Note, however, our main point that this can be interpreted as better evidence for an earlier theory of ratio, rather than proportion.

45 Propositions 1-3 of Book I consistute a complete theory of application of lines!

46 See $\$ 11$. 
the definition is stated, the said becomes immediately clear. For the areas and the bases have the same antanairesis; such is the definition of the same ratio.

In commenting on this passage, Alexander of Aphrodisias ${ }^{47}$ explains that the figure is a parallelogram, that 'similarly' means 'proportionately', and that

the said expressed in such-like terms is not familiar, but it becomes familiar once the definition of 'in proportion' is stated, that the line and the space are divided in proportion by the drawn parallel. Now this is the definition of proportionals which the ancients used: those magnitudes are in proportion to each other (and similar to each other) of which the anthyphairesis is the same. But he has called anthyphairesis antanairesis. ${ }^{48}$

Here we have Aristotle referring to the result occurring at VI, 1:

Triangles and parallelograms which are under the same height are to one another as their bases.

This proposition is the step needed to express the geometrical properties of similar plane ${ }^{49}$ figures in terms of ratio, and the most important of a small group of propositions needed to develop the whole of Book $\mathrm{X}^{50}$ Euclid proves it using the definitions of Book $\mathrm{V}$ by showing that $\mathrm{V}$, Definition 5 applies directly; Aristotle sketches in one line an earlier definition and proof; Alexander annotates, amplifies, and explains Aristotle's outline, including what might seem to be, judging by its context, a direct quotation from Eudemus' history: ${ }^{51}$ "those magnitudes are in proportion to each other of which the anthyphairesis is the same." And, as Aristotle observes, the idea of this key proposition is indeed immediately clear, since the operation of performing anthyphairesis on the two parallelograms generates the anthyphairesis of their bases, and vice-versa (Figure 4).

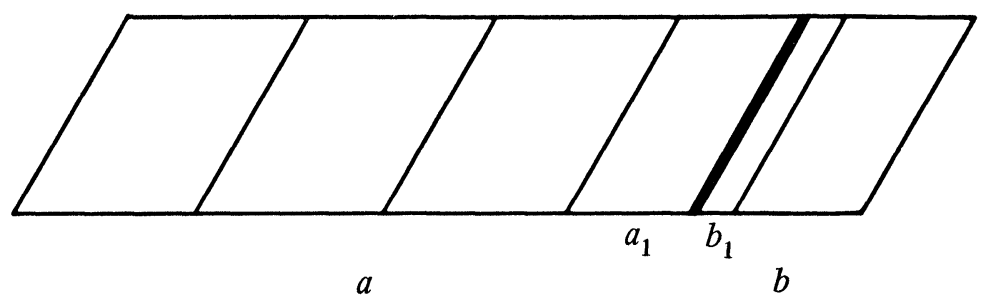

FigURE 4

47 In Topica, ed. M. Wallies, p. 545.

48 The parenthetic phrase is not in all surviving manuscripts. The etymology of the two words is roughly as follows:anthyphairesis = anto-hypo-hairesis, 'reciprocal sub-traction'; antanairesis = anti-ana-hairesis, 'reciprocal re-traction'. Nicomaches uses the further variation antaphairesis. See Knorr, EEE, p. 290 n. 26, for further details and references.

49 Note also how the same result for solids is stated by Euclid, at XI, 25, in precisely the Aristotelian form.

so See Knorr, $E E E$, pp. 259 and 262-270.

s1 Recall Eudemus' "summary style"; but see note 85 . 
The second kind of evidence is arithmetical. While we do not know how early Greek mathematicians performed their calculations, some of the results we do know indicate that they must have been using and understanding, in a practical way, techniques generating the convergents of a continued fraction expansion, ${ }^{52}$ a procedure closely related to anthyphairesis. Here are some examples.

(a) Aristarchus of Samos makes two assertions in On the Sizes and Distances of the Sun and Moon; ${ }^{53}$

'But 7921 has to 4050 a ratio greater than that which 88 has to 45 .

But 71755875 has to 61735500 a ratio greater than that which 43 has to 37.'

(b) Archimedes, in Measurement of a Circle $^{54}$ quotes without explanation the result that (paraphrasing in our notation)

$$
\frac{265}{153}<\sqrt{ } 3<\frac{1351}{780}
$$

(c) Hero, in his report of Archimedes' further calculations, Metrica $I^{55}$ says:

Archimedes proves in his work on plinthides and cylinders that of every circle the perimeter has to the diameter a greater ratio than 211875:67441, but a lesser ratio than 197888:62351. But since these numbers are not well-suited for practical measurements, they are brought down to very small numbers, such as 22:7.

(d) The side and diameter numbers give a procedure for generating successive approximations by ratios of numbers to the ratio of a side and diameter of a square. Such values were used by Aristarchus, where 7:5 is calculated as a lower bound for the ratio diameter:side, and by Hero, who uses 17:12.

(e) Knorr elucidates two further instances, in a Scholium to Euclid II, 11, and a passage from pseudo-Aristotles' Indivisible Lines. ${ }^{56}$

We shall explain the manipulations implicit in these examples in the next section and the Appendix. Further evidence of a different kind for anthyphairetic ratio theory will be considered in $\$ \$ 10$ and 11.

9. Arithmetical implications of anthyphairesis. At first sight, our proposals might seem inconsistent. We have argued that, on the one hand, manipulations of fractions were not admitted within the classical mathematical corpus, that any attempt to "cut up the 'one' " would be rejected by "the experts". On

52 This will be explained further in the next section and the Appendix.

53 On the sizes and distances of the sun and moon extracted in Thomas, SGM II, pp. 4-15.

54 Extractd in Thomas, SGM I, pp. 316-333.

55 Metrica 1, extracted in Knorr (1976) p. 115. The numbers occurring in this extract are corrupt:the alleged lower bound is in fact an upper bound, while the upper bound is a poorer approximation than 22:7, calculated by Archmimedes in his Measurement of $a$ circle. See Knorr, op.cit., for a discussion of interpretations and new proposals.

56 See Knorr, $E E E$, pp. 34-36. 
the other hand, we have arithmetical evidence of techniques for approximating ratios, apparently based on anthyphairesis, and these would therefore appear to require the manipulation of 'continued fractions', manipulations that would seem to presuppose the free use of ordinary fractions. ${ }^{57}$

To illustrate: the statement that the anthyphairesis of the diameter and side of a square is one, two, two, etc., can be translated into a statement about real numbers, that

$$
\sqrt{ } 2=1+\frac{1}{2+\frac{1}{2+\cdots}} .
$$

Truncating this before the successive addition signs and simplifying the expressions obtained gives the sequence of fractions

$$
\frac{1}{1}, 1+\frac{1}{2}=\frac{3}{2}, 1+\frac{1}{2+\frac{1}{2}}=\frac{7}{5}, \frac{17}{12}, \text { etc. }
$$

i.e. the quotients of successive side and diameter numbers. The other examples considered in the previous section can be treated in the same way. But how could such calculations be formulated and performed if fractional arithmetic was prohibited-not to mention the intrusion of real numbers? $?^{58}$

We shall now show that it is possible to perform these operations within the Greek context. At the outset I must emphasize that the algorithm described here is not proposed as a reconstruction of an original procedure; it is introduced only to demonstrate that the calculations are possible, and that one can illustrate, conjecture and prove many properties of anthyphairesis straightforwardly and naturally within the setting of the Elements.

We proceed by describing a problem, an algorithm ${ }^{59}$ for its solution and an illustration of its application:

Problem 1. Given a ratio $\theta$, find ratios of numbers $p: q$ approximating $\theta$, possibly with an indication of whether they are under- or overestimates.

Algorithm 1. If $p: q$ is an underestimate and $r: s$ is an overestimate for $\theta$, then $p+r: q+s$ will be a better approximation than that original estimate lying on the same side of $\theta$. Starting with $0: 1$ as underestimate and 1:0 as overestimate will thus generate a sequence of estimates of rapidly increasing accuracy. The terms occur in runs of under- and overestimates, and the estimates ending each run will be the best ones to consider. This will give an alternating sequence of under- and overestimates.

57 Some of the theory underlying anthyphairesis is developed in the Appendix, and some appreciation of this theory will be essential for an understanding of what follows. Continued fractions are considered in $§ \mathrm{~A} 3$.

58 These criticisms apply particularly to the constructions of Taylor (1926) and Thompson (1929).

59 This enunciation is arranged to coincide exactly with the version given in the Appendix, 8A2 except that $p$ and $q$, and $r$ and $s$ have been transposed. This means that the starting estimates chosen here would be highly implausible in a Greek context; but without change of substance and emphasis, they could be replaced by estimates of the type $n_{0}: 1$ and $n_{0}+1: 1$, where $n_{0}$ is the first term of the anthyphairesis. Since we are not claiming to give a literal reconstruction of an original procedure we shall not make this modification. Variants of this algorithm are discussed in Knorr (1976) pp. 136-139. 
This algorithm is based on the fact that if $p: q \leqslant r: s$, then $p: q \leqslant p+r: q+$ $s \leqslant r: s$. While such a result does not appear in Euclid, results very close to it are considered as a central part of proportion theory. For example, the result is proved in the case of equality: if $p: p^{\prime}:: q: q^{\prime}:: \ldots:: r: r^{\prime}$ then $(p+q$ $+\cdots+r):\left(p^{\prime}+q^{\prime}+\cdots+r^{\prime}\right):: p: p^{\prime}$. This occurs both in the older arithmetical form (VII, 12) and, with an almost identical enunciation, in a version relating to magnitudes $(\mathrm{V}, 12)$; and a similar proposition dealing with subtraction also occurs in both books (VII, 11 and V, 19). Inequalities between ratios are not considered in Book VII; they are handled in Book V, starting at Proposition 10 (where Euclid assumes, without justification, that ratios are totally ordered by size). The result we are using can now be derived immediately: paraphrasing, if $p: q:: x: s$, then $x \leqslant r$, so $p: q:: p+x: q+s \leqslant p$ $+r: q+s$, and the corresponding right hand inequality follows similarly. ${ }^{60}$

Some results of the algorithm are exhibited so strikingly in any example that they would clearly be worth investigating, though it seems improbable that any kind of general theory or formal verification of these properties would be within the scope of early Greek mathematicians. As an illustration, consider the following calculation of successive approximations to the ratio of the diameter and side of a square.

We start with diameter:side lying between $0: 1$ and 1:0, and these generate the new ratio 1:1; we find diameter:side lies between $1: 1$ and 1:0 and these generate 2:1 which is an overestimate, so diameter:side now lies between 1:1 and 2:1. Setting this out and continuing for a few steps we get:

diameter:side lies between

this generating a new

\begin{tabular}{cccc} 
underestimate & overestimate & underestimate & overestimate \\
\hline $0: 1$ & $1: 0$ & $1: 1$ & \\
$1: 1$ & $1: 0$ & & $2: 1$ \\
$1: 1$ & $2: 1$ & & $3: 2$ \\
$1: 1$ & $3: 2$ & $4: 3$ & \\
$4: 3$ & $3: 2$ & $7: 5$ & \\
$7: 5$ & $3: 2$ & & $10: 7$ \\
$7: 5$ & $10: 7$ & & $17: 12$ \\
etc. & & &
\end{tabular}

From this and other examples ${ }^{61}$ we see the following general patterns emerging:

60 Ian Mueller has pointed out that this argument is fallacious since it requires a fourth proportional to three given numbers, and this will not normally exist, e.g., as in $2: 3:: x: 4$. The argument can be maintained by passing to commensurable geometrical magnitudes; or it can be reworked so as to avoid this step, for instance by passing via an extension of VI, 16 that $p: q<r: s$ is equivalent to $p s<q r$. In any case, I am arguing here for the possibility of a kind of experimental arithmetical exploration that would be carried out without great regard, to begin with, for the formal justification of each step.

61 See Fletcher (1973) for many other examples illustrating approximating roots and logarithms, the diatonic and other scales with unequal or equal treatment, features of the calendar, etc. Taylor (1926) and Thomson (1929) also give examples and remark on some of the following characteristics in an interpretation of passages of Plato and Aristotle which, in mathematical terms, is closely related to our proposals. But their work is vitiated by their unremitting imposition of anachronistic procedures and preoccupations, without due consideration for the historical record. 
(a) The sequences of under- and overestimates generated by the algorithm converge rapidly towards each other.

(b) Under- and overestimates occur in runs, and the run-end estimates in this case are the side and diameter numbers.

(c) Alternate pairs of estimates satisfy simple relationships. For example, the successive run-end estimates always satisfy

$1 \times 1-1 \times 2=-1 ; \quad 3 \times 5-2 \times 7=+1 ; \quad 7 \times 12-5 \times 17=-1$; etc.

(d) The length of each run of under- and overestimates is the corresponding term in the anthyphairesis of the ratio, which therefore can be read off from the table; here Anth(diameter:side) $=[1,2,2,2, \ldots]$.

(e) If any ratio in the scheme is chosen, and the algorithm is performed on that ratio, the original calculation will be duplicated up to the chosen ratio, at which point it will terminate. The run-end ratios are particularly significant; restating this observation for them, in terms of anthyphairesis, as illustrated by this example, we see $\sqrt{ } 2: 1=[1,2,2,2, \ldots] ; 1: 1=[1] ; 3: 2=[1,2]$; $7: 5=[1,2,2] ; 17: 12=[1,2,2,2]$, etc. ${ }^{62}$

(f) The anthyphairesis of any incommensurable ratio of the form ${ }^{63} \vee p: \bigvee q$ with $p>q$ becomes periodic with the second term, and its periods exhibit a characteristic palindromic pattern:

$$
\vee p: \vee q=\left[n_{0}, n_{1}, n_{2}, \ldots, n_{2}, n_{1}, 2 n_{0}, n_{1}, n_{2}, \ldots, n_{2}, n_{1}, 2 n_{0}, \ldots\right]
$$

(g) The anthyphairesis of any ratio of the form $(p / q+V(r / s)): 1$ eventually becomes periodic, so can be calculated in a finite number of steps. ${ }^{64}$

(h) The algorithm applied to the reciprocal of a ratio is closely related to the algorithm applied to the ratio. For example, if $\operatorname{Anth}(A, B)=$ $\left[n_{0}, n_{1}, n_{2}, \ldots\right]$, and if $A<B$, then $n_{0}=0$ and $\operatorname{Anth}(B, A)=\left[n_{1}, n_{2}, \ldots\right]$; or if $A>B$, then $n_{0} \neq 0$ and $\operatorname{Anth}(B, A)=\left[0, n_{0}, n_{1}, n_{2}, \ldots\right]{ }^{65}$

(i) The connection between the ordering of ratios ${ }^{66}$ and their anthyphairesis has a complicating feature that is clearly illustrated by the following examples: If $n<m$ then $[n, \ldots]<[m, \ldots]$, while $[p, n, \ldots]>[p, m, \ldots]$, and $[p, q, n, \ldots]<[p, q, m, \ldots]$, and so on, alternately, whatever numbers $p, q$, etc. precede, and whatever different finite or infinite sequences succeed the first different entries $n$ and $m$.

Properties (a) to (e) will be proved and discussed in the Appendix, using an interpretation of this algorithm in cartesian coordinates due to H. J. S. Smith; (f) and (g) were proved by Euler, Lagrange, and Galois; (h) is obvious; and (i) can be deduced easily.

62 This shows explicitly the calculation of the convergents of the continued fraction expansion of the real number $\sqrt{ } 2$ is possible. Note also the symbolic use of the anthyphairetic definition of a ratio: 10:7 $=[1,2,2,2]$, etc.

63 By $\vee p: \bigvee q$ we mean the ratio (side of a square of area $p$ ):(side of a square of area $q$ ), constructed using II, 14, for example; see notes 1 and 90 . We shall always suppose hereafter that the two sides are incommensurable, and $p>q$.

64 This property is mentioned at this point for convenience; it is most unlikely to have been spotted by experiment. See $\$ 11$ for a further discussion.

65 The occurrance of $n_{0}=0$ in any application could therefore be avoided by reciprocating.

66 Here we follow Euclid in assuming, without further comment or proof, that ratios are totally ordered by size. 
If anthyphairesis, or an associated procedure, was established as a method for defining ratio, an obvious problem would suggest itself, but would lead to the following surprising negative conclusion:

(j) There appears to be no connection whatsoever between $\operatorname{Anth}(p, q)$, $\operatorname{Anth}(r, s)$, and $\operatorname{Anth}(p r, q s)$ or $\operatorname{Anth}(p r+q s, q s)$. In other words, anthyphairesis is only very tenuously connected with the addition and multiplication of fractions. ${ }^{67}$

This is a characteristic but surprising feature of anthyphairesis. The first algorithm on this problem, due to A. Hurwitz, dates from 1891 and only relates $\theta$ to $2 \theta$; and it is only relatively recently, with the increased understanding of algorithmic procedures arising from the interest in high speed computation, that simple general algorithms have been developed. ${ }^{68}$ The difficulty is a reflection of the deep and intractable feature that, apart from elementary properties of commensurable ratios and quadratic surds, and a few isolated results, almost nothing is known about the behaviour of the terms occuring in the anthyphairesis, even of a binomial like $(\sqrt{ } 2+\sqrt{ } 3): 1$, or the simplest cube root $\sqrt[3]{2}: 1$, the solution of the Delian problem, except that they can be calculated by simple arithmetical procedures. (For example, it is still not known whether the terms in these particular expansions are bounded, or normally distributed.) Gauss attempted to calculate the probability distribution functions of the terms of the anthyphairetic sequence but his first numerical calculations led him to write, on February 5, 1799, that "Tam complicatae evadunt, ut nulla spes superesse videatur". ${ }^{69}$ Nearly two years later, on October 25, 1800 he recorded in his celebrated notebook that "Problema e calculo probabilitatis circa fractiones continuas olim frustra tentatum solvimus" ${ }^{70}$ His success was only partial, however, as he describes in a letter ${ }^{71}$ to Laplace on January 30th 1812:

Je me rappelle pourtant d'un probleme curieux, duquel je me suis occupé il y a 12 ans, mais lequel je n'ai pas reussi alors à

67 See Khinchnin (1964), p. 20: "There is, however, another and yet more significant practical demand that the apparatus of continued fractions does not satisfy at all. Knowing the representations of several numbers we would like to be able, with relative ease, to find the representations of the simpler functions of these numbers (especially, their sum and product). In brief, for an apparatus to be suitable from a practical standpoint, it must admit sufficiently simple rules for arithmetical operations; otherwise it cannot serve as a tool for calculation. We know how convenient systematic fractions are in this respect. On the other hand, for continued fractions there are no practically applicable rules for arithmetical operations; even the problem of finding the continued fraction for a sum from the continued fraction representing the addends is exceedingly complicated, and unworkable in computational practice".

68 See A. Hurwitz (1891), M. Hall (1947), R. W. Gosper (1972) and G. N. Raney (1973). I am indebted to Mike Paterson for explaining these results lucidly to me and staging a demonstration of their operation with Meurig Beynon and a small programmable calculating machine. Here is one addition that they evaluated: $[1,1,1, \ldots]+[0,3]=$ $[1,1,19,1,1,3,1,1,19, \ldots]$.

69 "They come out so complicated that no hope appears to be left." See Gauss, Werke, vol. X, pp. 553-554.

70 "We are solving problems from the calculus of probabilities about continued fractions that once we attempted in vain." Op.cit., p. 552.

71 Op.cit., pp. 371-374. 
resoudre à ma satisfaction. Peut être daignerés vous vous en occuper quelques momens: dans ce cas je suis sur que vous trouverés une solution plus complete. Le voici. Soit $M$ une quantité inconnue entre les limites 0 et 1 , pour laquelle tou[te]s les valeurs sont ou egalement probables ou plus ou moins selon une loi donnée: qu'on la suppose convertie en une fraction continue

$$
M=\frac{1}{a^{\prime}+\frac{1}{a^{\prime \prime}+\text { etc. }}} .
$$

Quelle est la probabilité, qu'en s'arretant dans le developpement à un terme fini, $a^{(n)}$, la fraction suivante

$$
\frac{1}{a^{(n+1)}+\frac{1}{a^{(n+2)}+\text { etc. }}}
$$

soit entre les limites 0 et $x$ ? Je la designe par $P(n, x)$ et j'ai en supposant pour $M$ toutes les valeurs egalement probables

$$
P(0, x)=x ;
$$

$P(1, x)$ est une fonction transcendente dependante de la fonction

$$
1+\frac{1}{2}+\frac{1}{3}+\cdots+\frac{1}{x}
$$

que Euler nome inexplicable et sur la quelle je viens de donner plusieurs recherches dans un memoire presenté à notre societé des sc[iences] qui sera bientot imprimé. Mais pour les cas ou $n$ est plus grand, la valeur exacte de $P(n, x)$ semble intraitable. Cependant j'ai trouvé par des raisonnemens tres simples que pour $n$ infini on a

$$
P(n, x)=\log (1+x) / \log 2 .
$$

Mais les efforts que $\mathrm{j}$ 'ai fait lors de mes recherches pour assigner

$$
P(n, x)-\log (1+x) / \log 2
$$

pour une valeur tres grande de $n$, mais pas infinie, ont été infructueux.

Gauss never published his "raisonnemens tres simples", and the first proof of the assertion was published in 1928 by Kuz'min. ${ }^{72}$ Further developments of the problem are summarised in a comprehensive modern treatise on the theory of computation by $\mathrm{Knuth}^{73}$ and he concludes his section on anthyphairesis with:

In view of the historical importance of Euclid's method, it

72 See Khinchin (1964), pp. 71-83.

73 Knuth (1969), 84.5.3. 
seems fair to state that a determination of the asymptotic behaviour of $\tau_{n}$ [a parameter closely related to the number of terms in the anthyphairesis of a commensurable ratio] is the most important problem in the analysis of algorithms which is still unsolved. The world's most famous algorithm deserves a complete analysis!

Our proposal, in $\$ 11$, that Book X of the Elements may have been the first venture into this still uncharted jungle adds a new spice to the history of ancient mathematics.

Finally, the experience gained by experimenting with the algorithm leads to an important insight:

(k) The algorithm manipulates inequalities between ratios and this might suggest the theoretical tool, that it may be easier to prove (or define) equality between ratios by showing that inequality leads to a contradiction. ${ }^{74}$ Similarly the alternating run-end sequences might lead to the idea of the exhaustion proofs of Book XII.

While our algorithm shows that the development of arithmetical procedures for performing anthyphairesis was well within the technical capacity of Greek mathematics, it seems highly implausible that they would have been able to give general proofs of results like (c), (f), and (g), since it is difficult to conceive of such proofs without using the much later developments of algebra and induction. Particular cases are possible; for example all properties of the algorithm for the example given of $\sqrt{ } 2: 1$ can be verified using the geometrical analysis of the side and diameter given in the previous section. In fact the quotation from Proclus describing sides and diameters ${ }^{75}$ continues with

And this is proved graphically in the second book of the Elements by him [sc. Euclid],

followed by an almost verbatim quotation of II, 10; this proposition can be used to prove the properties. This example can be generalised to prove $\vee\left(n^{2}+1\right): 1=[n, 2 n, 2 n, \ldots]$; the result that $\vee 3: 1=[1,1,2,1,2, \ldots]$ can be proved geometrically and generalised two ways, to $\mathrm{V}\left(n^{2}-1\right): 1=[(n$ $-1), 1,2(n-1), 1,2(n-1), \ldots]$ and $V\left(n^{2}+2\right): 1=[n, n, 2 n, n, 2 n, \ldots]$. These results are all special cases of the yet more general result that $\mathrm{V}\left(n^{2} m+\right.$ $2 n): \vee m=[n, m, 2 n, m, 2 n, \ldots]$ which itself can be proved geometrically by elaborations of the same method, based on manipulating gnomons around squares in the style of Book II of the Elements. ${ }^{76}$ Ratios whose periods contain three terms, therefore of the form $[n, m, m, 2 m, m, m, 2 m, \ldots]$, can be evaluated by the same methods; but, beyond this, the technique rapidly

74 The reconstruction of a proportion theory intermediate between anthyphairesis and Book V, Definition 5, using inequalities and based on an examination of Archimedes' work, is described in Knorr (1977); an expanded version of this is to appear. A comparison of the formal detailed proofs of the propositions of proportion theory using anthyphairesis and V, Definition 5 is given in Appendix B of Knorr, EEE, pp. $332-344$. See $\$ 7$ for the beginning of the quotation.

76 The procedure is described in a forthcoming paper, Book II of Euclid's Elements and a pre-Eudoxan theory of ratio, (to appear). 
becomes too cumbersome and examples have to be treated case-by-case using different methods. ${ }^{77}$

10. Theoretical and practical logistic. We have argued that there was a well developed, though possibly informal, use of anthyphairesis to define ratio before the development of Book V-style proportion theory; we now propose that theoretical logistic (logistike) should be interpreted as the study of ratio during this period. This accords precisely with the usages we have considered in Plato, Archytas, and Aristotle, where it appears as an art closely related to geometry, in which important advances have recently been made, and which embodies the theoretical aspects of calculations. ${ }^{78}$ Let us check this interpretation against two further passages of Plato where descriptions of the scope of arithmetic and logistic are given. First, at Gorgias 451B:

Suppose some one asked me about one or other of the arts which I was mentioning just now: Socrates, what is the art of numeration (arithmetike)? I should tell him, as you did me a moment ago, that it is one of those which have their effect through speech. And suppose he went on to ask: With what is its speech concerned? I should say: With the odd and even numbers, and the question of how many units there are in each. And if he asked again: What art is it that you call calculation (logistike)? I should say that this also is one of those which achieve their whole effect by speech. And if he proceeded to ask: With what is it concerned? I should say-in the manner of those who draft ammendments in the Assembly - that in most respects calculation is in the same case as numeration, for both are concerned with the same thing, the odd and the even; but that they differ to this extent, that calculation considers the numerical values of odd and even numbers not merely in themselves but in relation to each other.

Arithmetic as the study of the odd and the even is a clear description of Pythagorean pebble arguments, ${ }^{79}$ but what, hitherto, could one make of the sense in which logistic is being used? This description must have had a clear meaning to Plato and his audience since we find it repeated almost word for word, in the Charmides (166A):

Thus reckoning (logistike), I suppose, is concerned with the

77 See van der Waerden, $S A$, pp. 144-146. Further discussion, with examples and references, are given in Knorr, $E E E$, pp. 118-126. General proofs of the results of this section, together with a table giving the anthyphairesis of $V p: 1$ for $2<p<50$, are given in Davenport (1968), pp. 77-113.

78 The statement that Theodorus is an expert in logistic (Statesman, 257A) and that he has taught the subject to Theaetetus (Theaetetus 145C-D) might seem at variance with our assigning the anthyphairetic definition of ratio to Theaetetus (see note 33). But the realisation that the procedure would constitute a definition of ratio would surely have to follow, perhaps as a late development, from familiarity with the use of the technique within geometry and number theory. (I am grateful to Myles Burnyeat for pointing out an error in an earlier version of this note.)

79 See Burkert, LS, pp. 427-438, and Knorr, EEE, pp. 131-169. 
even and the odd in their numerical relations to themselves and one another, is it not?

There are striking features of the anthyphairetic definition of ratio to which this could be referring. First the ordering by size of two ratios does not follow a simple lexicographical rule, as with expansions of numbers to some given base, but has this rule reversed in the odd numbered places of the anthyphairetic sequence ${ }^{80}$ - here we have "the even and the odd in their numerical relations to one another". Secondly, taking the successive run-end approximations generated by the anthyphairesis, we get increasingly accurate alternate under- and overestimates for a given ratio ${ }^{81}$ - "the even and the odd in their numerical relations to themselves"-and finally the arithmetical relationships like $823 \times 5-2 \times 7=+1$ give a further meaning for "to one another".83

It might be objected that these explanations are far too elaborate and complicated, and that Plato was referring to aspects of the study of odd and even that is preserved for us in the Elements at VII, Definitions 6-10 and IX, 21-34. But these are elementary propositions, deduced directly from straightforward definitions, and do not fit the description of "studies that demand more toil in the learning and practice we shall not discover easily nor find many of them" (Republic 526B; see §2). And further, all the features that we have been discussing - an independent definition of ratio, logistic as the study of ratio, the connection between logistic and geometry, the introduction of odd and even, and under- and overestimates-come together in two other long and important passages, from which we give short excerpts. At Republic 509D-510E, Plato develops an involved geometrical analogy of a line divided in a given ratio, with each section again subdivided in the same ratio, to represent the relation between visible and intelligible objects. Glaucon remarks that he cannot understand, and the passage continues:

Well, I will try again for you will better understand after this preamble. For I think you are aware that students of geome-

80 See $\$ 9$, paragraph (i).

81 See $\$ 9(a)$.

82 See $89(\mathrm{c})$.

83 Here is an additional way of differentiating between the even, the odd, and unity and elucidating "this trifling matter, of distinguishing one and two and three" (Republic 522C) using anthyphairesis:We have seen in $\$ 7$ that the anthyphairesis of the diagonal and side of a pentagon yields the sequence one, one, one, ...; and a slight modification of the first step the procedure for a square gives the anthyphairesis of side plus diagonal to side to be two, two, two, .... What about further such examples? It is indicated at the end of $\$ 9$ that it can easily be guessed from arithmetical studies and proved that $\left(n+V\left(n^{2}+1\right)\right): 1=[2 n, 2 n, 2 n, \ldots]$, and this is well within the scope of early Greek mathematics; but a vast amount of arithmetical exploration would be needed to produce a similar result whose terms are odd. In fact-as we now can work out algebraically-if $\theta: 1=[n, n, n, \ldots]$, then $\theta=\frac{1}{2}\left(n+\vee\left(n^{2}+4\right)\right)$, and the $\frac{1}{2}$ which here does not cancel out introduces unexpected complications into the calculations. To illustrate, $(3+\sqrt{ } 13): 1=[6,1,1,1,1,6, \ldots], \frac{1}{2} \vee 13: 1=[1,1,4,14,4,1,2, \ldots], \frac{3}{2}: 1$ $=[1,2]$, but $\frac{1}{2}\left(3+V^{13}\right): 1=[3,3,3, \ldots]$. Geometrically, the solution can be found as a fairly straightforward extension of the line divided in extreme and mean ratio; see the paper referred to in note 76 and a forthcoming paper $A$ generalisation of the golden section. 
try and reckoning (logistike) and such subjects first postulate the odd and the even and the various figures and three kinds of angles and other things akin to these in each branch of science, regard them as known, and, treating them as absolute assumptions do not deign to render any further account of them to themselves or others, taking it for granted that they are obvious to everybody.

And in the Protagoras (356A-357B) Plato develops an analogy with weighting, also introduced in the Charmides after the passage quoted above, considering the weighting of pleasure and pain against themselves and against each other. The procedure is described by an analogy with what is clearly logistic although this word is not explicitly used:

If the saving of our life depended on the choice of odd and even, and on knowing when to make a right choice of the greater and when of the less-taking each by itself or comparing it with the other, and whether near or distant-what would save our life? Would it not be knowledge; a knowledge of measurement, since the art here is concerned with excess and defect, and of numeration, as it has to do with odd and even?

The subsequent change from ratio theory to proportion theory also gives a possible explanation of the development of the meaning of logistic into practical calculation, and this would be worth investigating further. When ratio theory, based on anthyphairesis, was abandoned for Book V-style proportion theory, ${ }^{84}$ the interest in anthyphairesis as a mathematical procedure would greatly diminish, and the details of its erstwhile connection with ratio would be forgotten. ${ }^{85}$ The precise meaning of the word ratio would be abandoned, then forgotten-V, Definition 3 would set the seal on that-and all results stated in terms of ratio would be interpreted and proved using the technique of the powerful new proportion theory. For examples of this shift of emphasis both Aristotle's Topics passage and Hippocrates' Quadrature of Lunes refer to ratio, but Alexander's commentary and the interpolated passages in Simplicius recast these statements in proportion theory. ${ }^{86}$

84 Becker, Knorr van der Waerden point out difficulties in establishing the proofs of basic results in ratio theory-in particular, and most unexpectedly, in proving that $A: C=$ $B: C$ implies $A=B$. Knorr argues ( $E E E$, pp. 261-273 and 332-344) that this would lead to the changeover to Book V-type proportion theory.

85 Thus, in Euclid we find it only in VII, 1-3, X, 2-4, and as a technique to be applied very occasionally within proofs. One view of the consequences of its use, in a restricted form, in V, 8 is given in Zeeman (1974). This proposition stands out from its context, both in content and style; but Malcolm Brown believes on stylitic grounds that it comes from an older, pre-Euclidean source, contrary to what Zeeman asserts.

86 The passage in Alexander's commentary: "Those magnitudes are in proportion to each other of which the anthyphairesis is the same", which we tentatively attributed to Eudemus, would appear to contradict this, since we would also expect Eudemus to give the ancient ratio-theoretic definition. But, as Knorr observes (EEE, p. 230): “Alexander nowhere explicitly cites this work [Eudemus' History of Mathematics]; but his discussion of Hippocrates' quadrature suggests that materials from Eudemus did reach him via intermediaries. See Simplicius, In Physica, ed. H. Diels, p. 56-57." This introduces a possibility of corruption. 
Notwithstanding this, the terminology of ratios was retained, probably because of its convenience and didactic value but, as its anthyphairetic meaning was forgotten, it would come to be thought that only the ratio of two commensurable magnitudes could be defined, in terms of 'multiple, part, or parts'. Eventually, since the ratio of commensurable magnitudes is a ratio of numbers, logistic, or ratio theory, would be perceived only as the study of manipulations of fractions; ${ }^{87}$ and any vestigial connection with anthyphairesis that remained would be rejected because of the near impossibility of relating anthyphairesis with addition and multiplication. ${ }^{88}$ that

11. A new perspective on Book $X$. We have interpreted Pappus' testimony ${ }^{89}$

[Theaetetus] was in my opinion the chief means of establishing exact distinctions and irrefragable proofs with respect to [the commensurable and the incommensurable, the rational and the irrational continuous quantities]

as explicit evidence for assigning to Theaetetus the development of a procedure for defining ratio (logos) using anthyphairesis, and proposed that logistike was the name given to this theory. Now let us examine the associated usage of alogon, without ratio. The word is introduced in X, Definition 3:

With these hypotheses [i.e. the Definitions 1 and 2 of commensurable/incommensurable and commensurable/incommensurable in square], it is proved that there exist straight lines infinite in multitude which are commensurable and incommensurable respectively, some in length only, and others in square also, with an assigned straight line. Let then the assigned straight line be called rational (rheton), and let those straight lines which are commensurable with it, whether in length and in square or in length only, rational, but those which are incommensurable with it irrational (alogon).

Denote the rational, the assigned straight line, by $\rho$; then another line is called rational (rheton, expressible) if it is of the form ${ }^{90}(p / q) \rho$ or $\mathrm{V}(p / q) \rho$;

87 See the quotation from Proclus in \$2. The view that 'elements' were an aspect of the study of anthyphairetically defined ratios is developed in a forthcoming paper Equations, neusis-constructions, and Euclid's elements: An anthyphairetic interpretation.

88 Periodically the anthyphairetic approach would be rediscovered. For example, some Arab mathematicians of the 9th to 12th centuries proposed it as a simpler explanation of ratio than Euclid's (see Plooij (1950)), and in the 1930's Becker recast Book V in terms of it, basing his work on a close scrutiny of the historical record, but strongly influenced by his philosophy of mathematics.

See 85 for the full quotation.

90 We are here not using Heath's notation, described in his note to X, Definition 3 in Euclid, $E E$ III, pp. 11-12. Heath does not name 'the rational', the assigned straight line, and uses $\rho$ to refer to any rational line, i.e. any line commensurable in length or square with the rational. We reserve $\rho$ for the name of 'the rational', and hence any rational will be of the form $\vee k \rho$, where $k=p / q$. (Recall that $\vee k \rho$ is shorthand for the side of the square with area $k \rho^{2}$; see notes 1 and 63.) The reason for this is explained in note 92 . 
but the rest are not called arrheton, but alogon, without ratio. Why two words for the same idea? A possible answer may be that Euclid has here conflated two ideas, each leading to separate problems, neither of which fits naturally into the new Book $\mathrm{V}$ proportion theory. ${ }^{91}$ We have previously noted that there may have been philosophical problems with an anthyphairesis whose calculation could not be performed in a finite number of straight-edge and compass constructions; let us suppose that the procedure was prohibited in such cases so that, for example, the binomial ${ }^{92}(\sqrt{ } 2 \rho+\sqrt{ } 3 \rho)$ was without ratio, alogon, with respect to $\rho$. We are now confronting a problem analogous to that facing the first discoverers of incommensurability, ${ }^{93}$ but from the position of greatly increased confidence bestowed by a conceptual framework within which the problem can be analysed and turned into a fruitful and creative subject of study: to develop a new technique for handling the alogoi. The result is a huge research programme of examining lines which arise from manipulations of the assigned line $\rho$, so see which lead to lines which are alogon with respect to previously constructed lines, and then to describe them by relating them to their construction out of segments with logos; this is what we find in Books X and XIII, ${ }^{94}$ and which is also possibly what Proclus was referring to when he wrote ${ }^{95}$ of the

great and unlimited complexity, such as the material that Apollonius has elaborated at considerable length about unordered irrationals.

In this description, however, we have presumed a solution of, or at least a working hypothesis concerning, the preliminary problem of characterising those ratios which have a logos, and this is where the rheton enters the argument. As we observed in $\S 8(f)$, the striking pattern of the anthyphairesis of a ratio of the form $\bigvee p \rho: \bigvee q \rho$ can be guessed from an arithmetical analysis well within with scope of Greek mathematics; but a general proof of this result, and the further result characterising ratios whose anthyphairesis be-

91 The translation of the different words into the same English root and the choice of a word with a closely related but different meaning do not help our understanding. The proposal by van der Waerden ( $S A$, p. 169) to translate rhetos as 'expressible', and alogos as 'unreasonable' would be a great improvement.

92 Heath's notation, which allows him to write a binomial as $\rho+\sqrt{ } k \rho$, blurs the distinction between lines, in our notation, of the form $\vee k \rho+\sqrt{ } 1 \rho$ and $k \rho+\sqrt{ } 1 \rho$. Since Euclid's practice in Book $\mathbf{X}$ is to regard the definitions as applying to the general case only, this second line does not have a name, even within the subdivision of binomials given in Proposition 48-53 and their associated Definitions, and so does not fall within the classification of Book X.

93 Cf. Gorgias (465A): "And I refuse the name of art to anything irrational."

94 See Knorr, $E E E$, p. 278: "In Elements XIII we encounter the proofs of the irrationality of several lines produced in association with the construction of the regular solids. To a modern reader, these applications of the theory of irrationals seem disingenuous; commentators take note of these theorems, to be sure, but they find little of significance in them". Then (pp. 279-284) he gives a new interpretation of Proclus' testimony that Eudoxus "by means of analysis increased in number the theorems about the section which took their start from Plato", based on the analysis of the magnitudes arising in the construction of an icosahedron in a sphere whose radius is the rheton.

95 Proclus' Commentary p. 74 (Friedlein's pagination); the work by Apollonius to which he refers is now lost. See also Pappus Pt. I, par. 1 (quoted in \$5). 
comes periodic and so is finitely computable (i.e. the unnamed set of ratios of the form $(p / q) \rho+\bigvee(r / s) \rho: \rho$ - see $\S 8(\mathrm{~g}))$ would seem to be beyond their techniques of proof. ${ }^{96}$ The set of the rheta then represents an intermediate vantage point; even if it were not possible to prove the result that $\bigvee p \rho: \bigvee q \rho$ always had an almost palindromic form, it would be reasonable to proceed with the development of the subject on the hypothesis that this was the case, separating off the implications of such a hypothesis from the problem of its proof, ${ }^{97}$ and leaving the question of general periodic anthyphairesis for later consideration.

In this connection, consider further the final passage of the quotation from Pappus:

It was Theaetetus who distinguished the powers which are commensurable in length from those which are incommensurable (i.e. in length) ... as is stated by Eudemus the Peripatetic.

(The word 'power', clearly coming from the Greek dynemis, is often mistranslated as 'square root' or 'surd' in such contexts. Its meaning is unambiguously 'second power': two 'powers', or squares, are commensurable 'in power' when their areas are commensurable; and commensurable 'in length' when their linear measurement, e.g. their sides or their diagonals, are commensurable. ${ }^{98}$ ) This passage is usually taken as a reference to $\mathrm{X}, 9$-a characterisation relating the ratio of two commensurable lines to the ratio of their squares - and this proposition is attributed to Theaetetus by a scholiast. However there is an objection to this interpretation, that Theodorus' lesson ${ }^{99}$ in Plato (Theaetetus 147C-148B) in which Theaetetus is described as developing his ideas on incommensurable roots finishes with an explicit indication that Theaetetus realises that this type of argument will apply to third powers as well:

\section{And a similar thing holds for solids}

and so is not characteristic of the dynemis, the second power. A slight textual

96 Could the disorismi be connected with the problem? See Proclus' Commentary pp. 66-68 (Freidlein's pagination), extracted in Thomas, SGM I, pp. 144-161: "Younger than Leodamus were Neoclides and his pupil Leon, who added many things to those known before them ... ; he [Leon] also discovered diorismi, showing when the problem investigated can be solved and when not". Proclus also places Leon chronologically between Theaetetus and Eudoxus.

97 This is now standard practice within mathematics. To give current examples: in number theory, the Riemann hypothesis is still unresolved, though in algebraic geometry, the Weil conjectures have been proved; and in functional analysis, the basis problem now has a counterexample.

98 See Knorr, $E E E$, pp. 65-69 for a full discussion of dynamesis. A similar confusion occurs with our use of 'square': to 'square a rectangle' (II, 14) means, in effect, to construct the square root of its area.

99 The first half of Knorr, $E E E$, is devoted to a discussion of the early theory of incommensurability and deals in detail with this passage. His Chapter III examines the text closely and sets up interpretive criteria; Chapter IV reviews all previous reconstructions of Theodorus' lesson; and Chapter VI proposes a new reconstruction. The later contributions of Theaetetus and Eudoxus are analysed in the second half of the book. 
amendment ${ }^{100}$ introduces an alternative interpretation, not open to this objection:it is possible to 'distinguish the lengths which are commensurable in power from those which are incommensurable (i.e. in power)' by anthyphairesis, since the former are those exhibiting the striking, almost palindromic, pattern $\left[n_{0}, n_{1}, n_{2}, \ldots, n_{2}, n_{1}, 2 n_{0}, n_{1}, n_{2}, \ldots, n_{2}, n_{1}, 2 n_{0}, \ldots\right]$ described in $\S 9(f)$, and precisely such a distinction is the purpose of $X$, Definition 3, between the rheton and the alogon. Support for such a reading is provided later in the Commentary, where we read (Part II, Paragraph 17) that:

Those who have written concerning these things (i.e. of irrationals), declare that the Athenian, Theaetetus, assumed two lines commensurable in square and proved that if he took between them a line in ratio according to geometric proportion (the geometric mean), then the line named the medial was produced, but if he took the line according to harmonic proportion (the harmonic mean), then the apotome was produced. We accept these propositions, since Theaetetus enunciated them.

This gives a clear indication, based on ancient authority, that Theaetetus started his classification from the dichotomy of commensurable in square /incommensurable in square, and not the distinction commensurable in length/incommensurable in length given by $\mathrm{X}, 9$.

The aim of this section has been to put forward a new type of explanation of the motivation for the classification of irrationals of Book X. The new insights into pre-Euclidean mathematics developed by Knorr follow from recognising and pursuing the inescapable fact that the construction and classification of incommensurables was a problem of central importance during the fourth century; but the impulse behind this problem remained unexplained. ${ }^{101}$ We have shown how it can be interpreted as an attempt to resolve an easily stated, credible, surprising, and central feature of anthyphairesis. But, with the development of Book V proportion theory, the urgency of the problem disappeared, and its statement became artificial; even the distinction between commensurable and incommensurable in length is not made in Book V, and when it is considered in $\mathrm{X}, 5$, the proof is flawed because Euclid fails to invoke Book V proportion theory. ${ }^{102}$ Simon Stevin remarked on this lack of motivation 400 years ago: ${ }^{103}$

100 I propose this amendment in ignorance of its plausibility in the sequence of translations and transcriptions through which the manuscript has passed. We have argued in $\$ 10$ that the early anthyphairetic foundation of mathematics was forgotren, and that this process was hastened by the influence of the Elements; hence a misunderstanding of Eudemus by Pappus is another possible source of error. J. P. Hodendijk has assured me that there is absolutely no justification in the Arabic text for making this amendment. Cf. Knorr, $E E E$, p. 4:"On the basis of one assumption I will find it possible to perceive the motives for the foundational work within the Greek mathematical discipline itself. This assumption is that the study of incommensurable magnitudes was a field of interest in its own right."

102 See \$3.

103 Quoted in Euclid, EE, III, pp. 8-9; but see J. Klein (1968), pp. 186-197 for a discussion of Stevin's bias against Greek mathematics. 
La difficulté du dixiesme Livre d'Euclide est à plusieurs devenue en horreur, voire jusque à l'appeler la croix des mathématiciens, matière trop dure à digérer, et en la quelle n'aperçoivent aucune utilité.

The only other development since Stevin's time is that the contents of Book X have been translated into algebra and forced into a Procrustean bed of the solution of special types of fourth-degree equations. Any alternative explanation to this would be worth some consideration; and one that arises directly out of the earlier proportion theory is surely worth a close scrutiny.

\section{Appendix: Approximaton by rational numbers, and continued fractions.}

\section{A 1. Introduction.}

If with a pair of rectangular axes in a plane we construct a system of unit points (i.e. a system of points of which the coordinates are integral numbers), and draw the line $y=\theta x$, we learn from the theorem that if $(x, y)$ be a unit point lying nearer to that line than any other unit point having a less abscissa (or, which comes to the same thing, lying at a less distance from the origin), $y / x$ is a convergent to $\theta$; and, vice versa $\hat{a}$ if $y / x$ is a convergent, $(x, y)$ is one of the 'nearest points'. Thus the 'nearest points' lie alternately on opposite sides of the line, and the double area of the triangle, formed by the origin and any two consecutive 'nearest points', is unity.

Thus H. J. S. Smith (1876) described a vivid geometrical interpretation of the continued fraction algorithm for finding best rational approximations (and, in the final paragraph of his paper, a method of solving the 'Diophantine' equation $p x-q y= \pm 1$ in integers). F. Klein (1908), regretting that continued fractions were only taught occasionally in schools, urged an extension of this approach on his audience. Recently T. Fletcher (1973) has simplified and brought it again to the attention of teachers. Here again I would like to explain the procedure, since it demonstrates vividly the theory lying behind anthyphairesis.

I shall proceed by describing three algorithms, and show how they are related. ${ }^{104}$

\section{A 2. Approximating by rationals.}

PROBLEM 1. Given a real number $\theta$, find rational numbers $q / p$ approximating $\theta$, possibly together with an indication of whether they are under- or overestimates. For example $\sqrt{ } 2 \div 17 / 12$ and $71755875 / 61735500>43 / 37$. For convenience, we only consider positive numbers $\theta$.

In the integer lattice (Smith's 'unit points') draw the line $y=\theta x$; we look for lattice points such that the line joining them to the origin has slope close to $\theta$. We can identify these lines by their end-points; the point $A=(p, q)$ will correspond to the line $y=q x / p$, and the approximation $q / p$ (see Figure 1), and we distinguish $q / p$ from its other representations $2 q / 2 p$, etc.

104 The first algorithm has already been considered, in an arithmetical form, in $\$ 9$. 


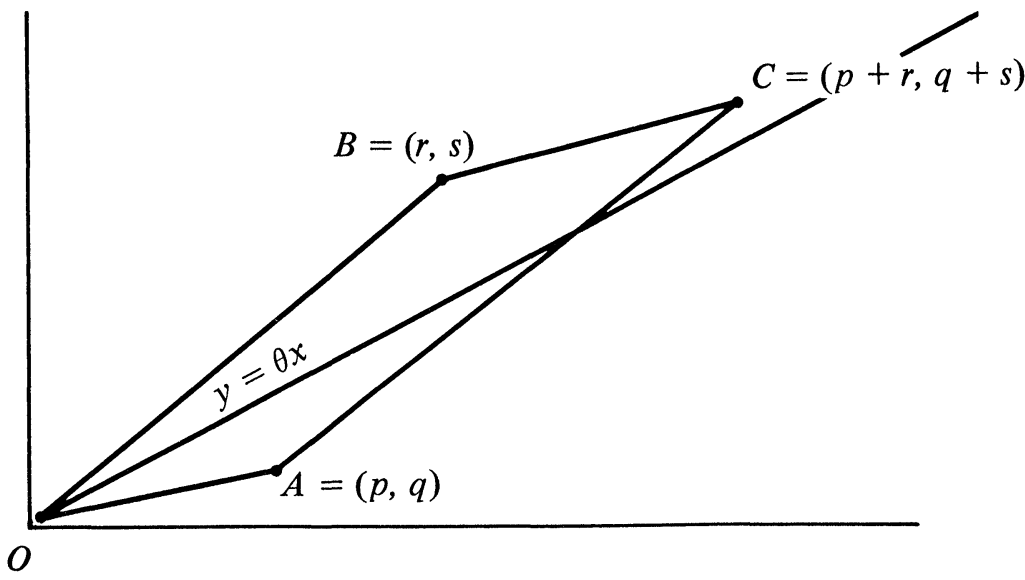

\section{FIGURE 1}

Algorithm 1. If $(p, q)$ is an underestimate and $(r, s)$ is an overestimate for $\theta$, then $(p+r, q+s)$ will be a better approximation than that original estimate lying on the same side of $y=\theta x$. Start with $(1,0)$ as underestimate and $(0,1)$ as overestimate, and thus generate a sequence of rational approximations to $\theta$.

EXAMPLE. When $\theta=\sqrt{ } 3$, we have $q / p \lessgtr \sqrt{ } 3$ according as $3 p^{2} \lessgtr q^{2}$. The algorithm generates the sequence (illustrated in Figure 2):

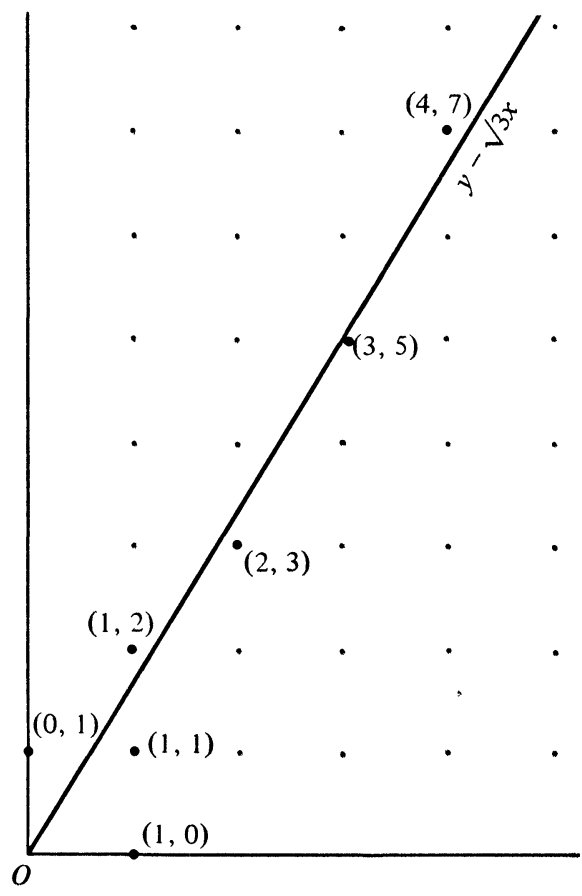

\begin{tabular}{cc} 
under & over \\
\hline$(1,0)$ & $(0,1)$ \\
$(1,1)$ &
\end{tabular}

etc.

FiguRE 2

At each stage, the vector sum of the current estimates is taken (e.g. $(2,3)+$ $(1,2)=(3,5))$; it is tested $\left(5^{2}<3.3^{2}\right.$, so it is an underestimate) and allocated to the appropriate column where it becomes the new estimate. 
Note how, if $(p, q)$ and $(r, s)$ are the current estimates, then $p \leqslant r$ and $q \leqslant s$, or $p \geqslant r$ and $q \geqslant s$, where both inequalities are strict, the step after the starting estimates have been superseded. Thereafter any estimate lies above and to the right of any earlier estimate.

The algorithm works quickly, directly, and easily in any situation where the test for an over- or underestimate can be applied, for example with roots and logarithms. ${ }^{105}$ To analyse it, we use the following result. ${ }^{106}$

PICK's THEOREM. The area of a simple polygon whose vertices are lattice points is (half of the number of points on the perimeter + the number of points inside - 1).

If $A$ and $B$ are the current estimates, generating the new estimate $C$ (see Figure 1), call $O A B$ the approximating triangle and $O A C B$ the approximating parallelogram.

PROPOSITION 1. The approximating triangle and parallelogram contain no points of the integer lattice.

Proof. Each step of the algorithm replaces the approximating triangle $O A B$ by $O A C$ or $O B C$, and each of these triangles is half of the approximating parallelogram $O A B C$; so the area of the approximating triangle is unchanged, and it starts with $A=(1,0), B=(0,1)$ and area $\frac{1}{2}$. Since 3 lattice points are vertices, and it has area $\frac{1}{2}$, it can have no lattice points inside.

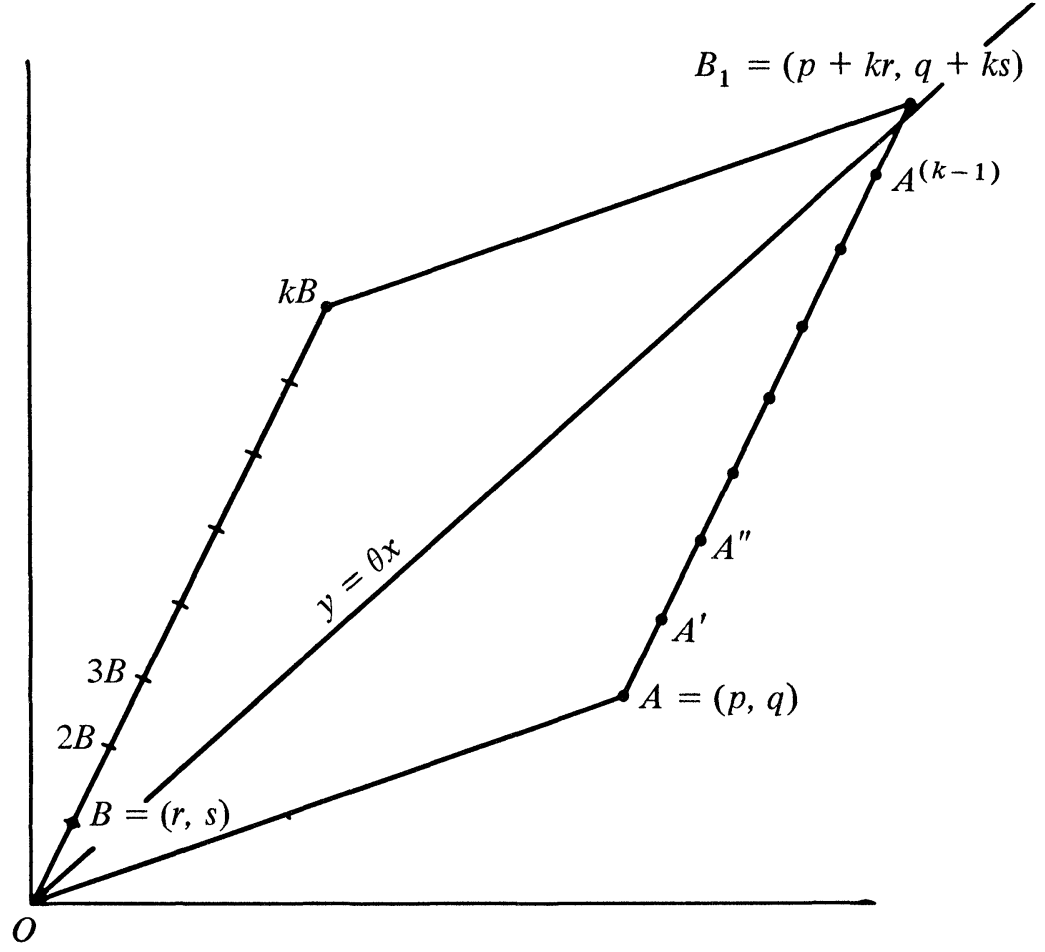

FIGURE 3

105 See note 61.

106 For proof, see, for example, Coxeter (1961), pp. 209-210. 


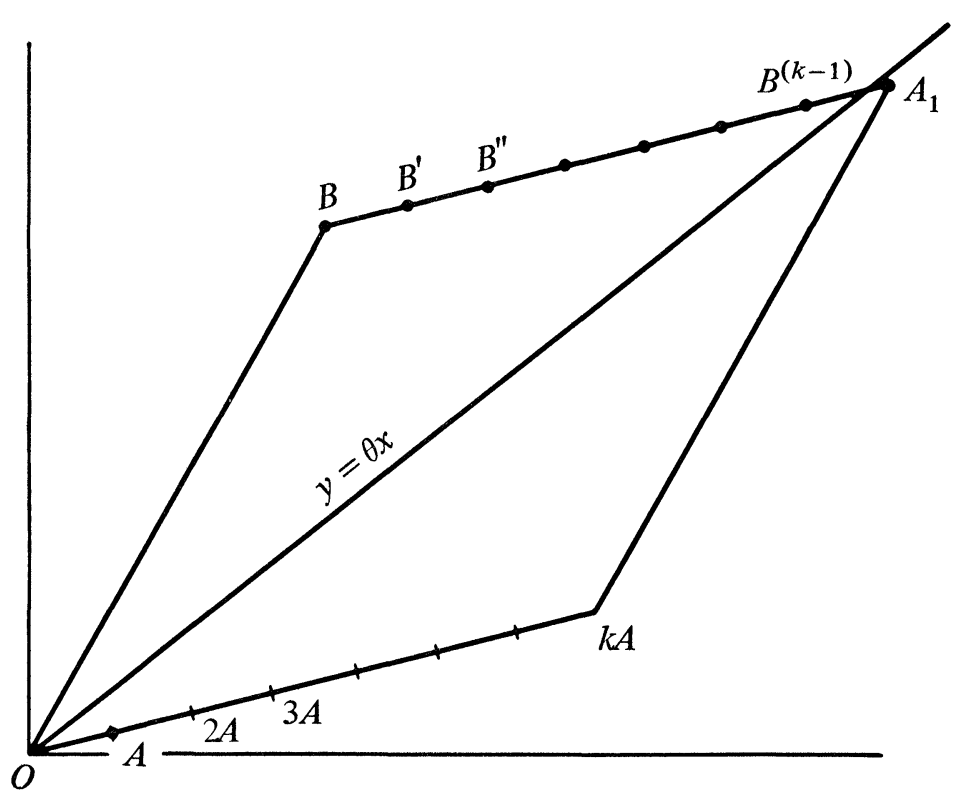

FIGURE 4

(Note: The thickness and areas of the parallelograms in all of the figures, except Figure 2, are exaggerated for clarity.)

A characteristic sequence of steps of the algorithm is illustrated in Figure 3: the estimates $A=(p, q)$ and $B=(r, s)$ give rise to a run of underestimates $A, A^{\prime}, \ldots, A^{(k-1)}$, followed by a new overestimate $B_{1}$. Each of the triangles $O A A^{\prime}, O A^{\prime} A^{\prime \prime}, \ldots, O A^{(k-1)} B_{1}$, being half of their corresponding parallelograms, will contain no lattice point. Hence neither the triangle $O A B_{1}$, nor $O B_{1}(k B)$ - which has the same area and the same number of perimeter lattice points-will contain any lattice points. Similarly none of the parallelograms in the tessellation determined by $O A B_{1}(k B)$ will contain any lattice points. The alternative situation, where $A$ and $B$ give rise to a run of overestimates $B, B^{\prime}, \ldots, B^{(k-1)}$ is illustrated in Figure 4.

COROllary 1.1. If $A=(p, q)$ is an underestimate, then there is no better underestimate with denominator $\leqslant p$. A similar result holds for overestimates.

Proof. Let $B$ be the current overestimate, and $B_{1}$ the first subsequent overestimate, preceded by the sequence $A, A^{\prime}, \ldots, A^{(k-1)}$ of underestimates (see Figure 3, and Figure 4 for the easier case $k=1$ ). Then $O A B_{1}$ contains no lattice points, and it also contains the triangle determined by $q / p \leqslant y \leqslant \theta x$ and $x \leqslant p$. The absence of a lattice point in this latter triangle shows that there is no better underestimate than $q / p$ with denominator not greater than p.

PRoposition 2. If $q / p$ and $s / r$ are the current estimates for $\theta$, then their errors are less than $1 / p r$, and one of these estimates will have error less than $1 / 2 p r$. 
Proof. The error in each estimate is less than

$$
\begin{aligned}
\left|\frac{s}{r}-\frac{q}{p}\right| & =\left|\frac{p s-q r}{p r}\right|=\frac{1}{p r}\left|\operatorname{det}\left[\begin{array}{lll}
1 & 0 & 0 \\
1 & p & q \\
1 & r & s
\end{array}\right]\right| \\
& =\frac{2}{p r} \times \text { area of approximating triangle }=\frac{1}{p r} .
\end{aligned}
$$

Since $\theta$ lies between $q / p$ and $s / r$ it will differ by less than $1 / 2 p r$ from the closest.

The approximations occur in runs of under- and overestimates, as illustrated in Figures 3 and 4. Call those estimates occuring at the ends of runs (e.g. $A^{(k-1)}$ in Figure $3, B^{(k-1)}$ in Figure 4 , and $5 / 3$ for $\sqrt{ } 3$ in the example) 'run-end estimates'.

COROLlaRY 2.2. If $q / p$ is a run-end estimate, its error is less than $1 / p^{2}$, and one of every pair of consecutive run-end estimates has an error less than half of this.

Proof. The next step gives an estimate $s / r$ with $r>p$.

Corollary 2.3. If $q / p$ is a run-end estimate, and the subsequent run is $n$ steps long, its error is less than $1 / n p^{2}$.

Proof. The subsequent run-end estimate $s / r$ has $r>n p$.

Hence the algorithm generates the best possible rational approximations to the slope $\theta$ for a given range of denominators-in other words, it minimises $|q / p-\theta|$-and we see that run-end estimates preceding long runs are likely to be particularly good.

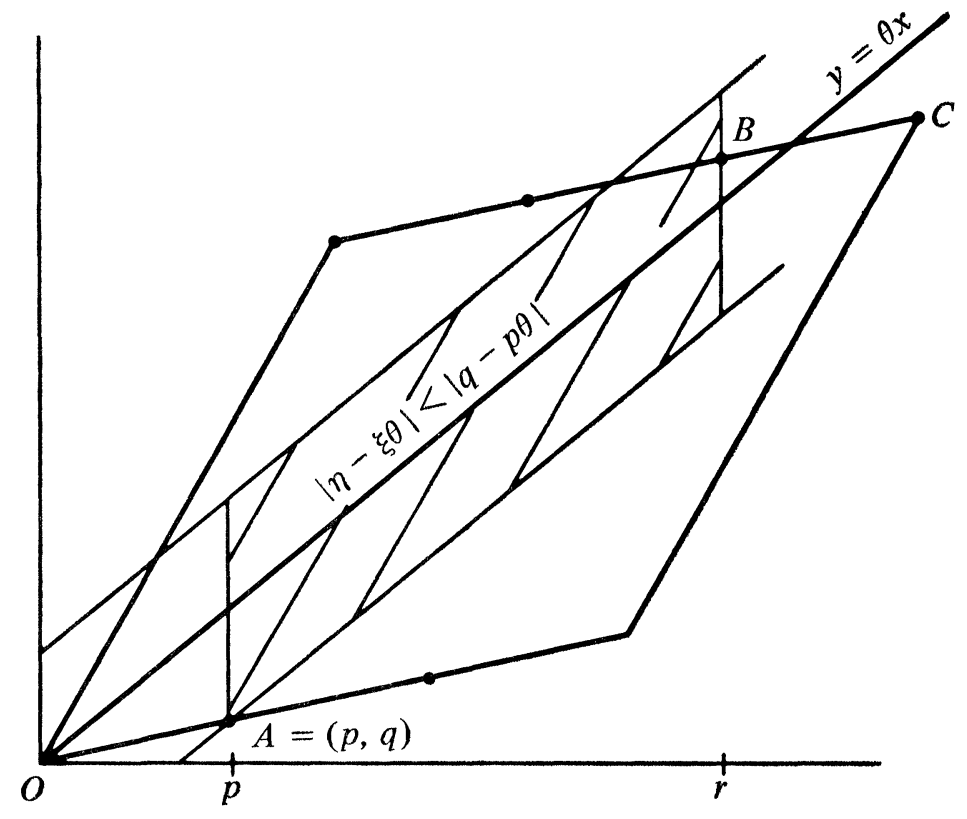

FiguRE 5 
The theorem referred to by Smith minimises $|p \theta-q|$ over a different range of $p$ 's; this corresponds to the different problem of finding successive points $(p, q)$ lying closest to the line $y=\theta x$. The result is as follows:

Proposimon 3. If $A=(p, q)$ and $B=(r, s)$ are consecutive run-end estimates, with $p<r, q<s$, then $|q-p \theta|<\left|q^{\prime}-p^{\prime} \theta\right|$ for $p^{\prime}<r$ and all $q^{\prime}$.

Proof. Consider the case illustrated in Figure 4 , where $B^{(k-1)}$ is now relabelled $B$ (see Figure 5). The set $(\xi, \eta)$ with $|\eta-\xi \theta|<|q-p \theta|$ will be a strip centered on $y=\theta x$ with $A$ on its boundary; it will contain $B$, and no other lattice point the range $p \leqslant p^{\prime}<r$. Now, applying a similar argument to the left of $A$, we see that the strip will contain no lattice point preceding $A$ (i.e. with $p^{\prime}<p$ ). Hence $A$ will be the closest lattice point to $y=\theta x$ in the range $0 \leqslant p^{\prime}<r$.

Since the run-end estimates lie on alternate sides of $y=\theta x$, we get:

Corollary 3.1. The successive closest lattice points to $y=\theta x$ occur on alternate sides.

A 3. Continued fractions. The simplest kind of continued fraction-all that we need consider-is an expression

$$
n_{0}+\frac{1}{n_{1}+\frac{1}{n_{2}+\ldots}},
$$

where all the denominators $n_{i}$ are positive integers. Truncating the expression at each step gives the successive convergents

$$
n_{0}, n_{0}+\frac{1}{n_{1}}, n_{0}+\frac{1}{n_{1}+\frac{1}{n_{2}}}, \text { etc., }
$$

a sequence of rational numbers. In a nonterminating continued fraction the limit of this sequence-if it exists-is the number represented by the fraction and the fraction is the expansion of this number; it will soon become evident that the sequence always converges when the $n_{i}$ are positive integers. For convenience, write a continued fraction as

$$
n_{0}=\frac{1}{n_{1}+} \frac{1}{n_{2}+} \ldots \text {, or }\left[n_{0}, n_{1}, n_{2}, \ldots\right] \text {. }
$$

Problem 2. Given a real number $\theta$, expand it as a continued fraction.

Algorithm 2. Write $\theta$ as an integer part and a fractional remainder, $\theta=n_{0}+\theta_{1}$. If $\theta_{1} \neq 0$, repeat with $1 / \theta_{1}: 1 / \theta_{1}=n_{1}+\theta_{2}$, and continue, $1 / \theta_{2}=n_{2}+\theta_{3}$, etc.

If we neglect the remainder $\theta_{k+1}$, so $\theta_{k} \doteqdot 1 / n_{k}$ then, substituting back, we get

$$
\theta \div n_{0}+\frac{1}{n_{1}+} \cdots \frac{1}{n_{k}}
$$

thus the convergents provide approximations to $\theta$. If any $\theta_{k+1}=0$, then $\theta$ is 
given by a terminating continued fraction, which is clearly a rational number; conversely, it is easily seen that if $\theta$ is rational, the algorithm terminates, since two steps reduce the size of both numerator and denominator of the $\theta$ 's.

Some examples will illustrate the algorithm.

(a) $\theta=\sqrt{ } 2=1+(\sqrt{ } 2-1)$ where $0<(\sqrt{ } 2-1)<1,(\sqrt{ } 2-1)^{-1}=\sqrt{ } 2$ $+1=2+(\sqrt{ } 2-1)$. So $\theta_{1}=\theta_{2}=\cdots=\sqrt{ } 2-1 ; n_{1}=n_{2}=\cdots=2$,

$$
\mathrm{V} 2=1+\frac{1}{2+} \frac{1}{2+} \frac{1}{2+} \ldots \text {, }
$$

and this has convergents $\frac{1}{1}, \frac{3}{2}, \frac{7}{5}, \frac{17}{12}, \frac{41}{29}$, etc. The denominators and numerators here are the successive side and diameter numbers. ${ }^{107}$

(b) $\frac{1}{2}(\sqrt{ } 5+1)=1+\frac{1}{2}(\sqrt{ } 5-1) ;\left(\frac{1}{2}(\sqrt{ } 5-1)\right)^{-1}=\frac{1}{2}(\sqrt{ } 5+1)=1$ $+\frac{1}{2}(\sqrt{ } 5-1)$. So

$$
\frac{1}{2}(\sqrt{ } 5+1)=1+\frac{1}{1+} \frac{1}{1+} \frac{1}{1+} \ldots,
$$

with convergents $\frac{1}{1}, \frac{2}{1}, \frac{3}{2}, \frac{5}{3}$, etc.. This, the 'golden number', is the number with the simplest nonterminating continued fraction and the poorest approximation by its convergents, the quotients of successive Fibonacci numbers.

These examples illustrate simple cases of a theorem of Lagrange, that the continued fraction of anything of the form $(p+\vee q) / r(p, q$, and $r$ integers) will eventually be periodic, and the periods of $\vee q$ will all begin with the second term. High-order roots need to be evaluated step by step; the procedure is easy, but this time no general formula for the $n_{i}$ can be derived. For example:

(c)

$$
\sqrt[3]{2}=1+\frac{1}{3+} \frac{1}{1+} \frac{1}{5+} \frac{1}{1+} \frac{1}{1+} \cdots
$$

with convergents $\frac{1}{1}, \frac{4}{3}, \frac{5}{4}, \frac{29}{23}, \frac{34}{27}$, etc.

(d) The continued fraction expansion of $\pi$ can be calculated by finding a sufficiently good rational (or decimal) approximation, and expanding that as a continued fraction. This gives

$$
\pi=3+\frac{1}{7+} \frac{1}{15+} \frac{1}{1+} \frac{1}{292+} \frac{1}{1+} \frac{1}{1+} \ldots,
$$

with convergents $3, \frac{22}{7}, \frac{333}{106}, \frac{355}{113}, \ldots$.

If Algorithms 1 and 2 are both performed on the same number, it becomes immediately apparent that they are very closely related. Specifically, if the geometrical procedure generates the sequence of run-end estimates $[0 / 1,1 / 0], q_{0} / p_{0}, q_{1} / p_{1}, q_{2} / p_{2}, q_{3} / p_{3}, \ldots$ for $\theta$, then $p_{k} / q_{k}$ is the $k$ th convergent of the continued fraction expansion $\theta$,

$$
\theta=n_{0}+\frac{1}{n_{1}+} \frac{1}{n_{2}+} \ldots ;
$$

the denominator $n_{k}$ is the run-length preceeding the run-end estimate $q_{k} / p_{k}$, and so the error in truncating at the $k$ th convergent is $\leqslant 1 / n_{k+1} p_{k}^{2}$; even $k$ 
gives an underestimate, odd $k$ gives an overestimate; and alternate errors decrease monotonically to zero, so the convergents do converge to $\theta$. Truncating before a large denominator should give particularly good approximations (e.g. $22 / 7$ and $355 / 113$ for $\pi$ ).

These results follow from the following result which is suggested by an examination of Figures 3 and 4.

Proposition 4. Writing the convergents of

$$
n_{0}+\frac{1}{n_{1}+} \frac{1}{n_{2}+} \ldots
$$

as

$$
\frac{q_{0}}{p_{0}}=\frac{n_{0}}{1}, \frac{q_{1}}{p_{1}}=\frac{n_{1} n_{0}+1}{n_{1}}, \frac{q_{2}}{p_{2}}, \ldots
$$

then

$$
\frac{q_{k+1}}{p_{k+1}}=\frac{n_{k+1} q_{k}+q_{k-1}}{n_{k+1} p_{k}+p_{k-1}}
$$

Proof. We proceed by induction. The case $k=1$ follows immediately from evaluating $q_{2} / p_{2}$. The general case follows by observing that

$$
\begin{aligned}
\frac{q_{k+1}}{p_{k+1}} & =n_{0}+\frac{1}{n_{1}+} \ldots \frac{1}{n_{k}+} \frac{1}{n_{k+1}} \\
& =n_{0}+\frac{1}{n_{1}+} \cdots \frac{1}{m} \quad\left(\text { where } m=n_{k}+\frac{1}{n_{k+1}}=\frac{n_{k+1} n_{k}+1}{n_{k+1}}\right) \\
& =\frac{m q_{k-1}+q_{k-2}}{m p_{k-1}+p_{k-2}} \quad \text { (by the inductive hypothesis) } \\
& =\frac{n_{k+1} q_{k}+q_{k-1}}{n_{k+1} p_{k}+p_{k-1}} \quad \text { (simplifying and using the inductive }
\end{aligned}
$$

hypothesis again).

REMARK. For the sake of clarity and vividness, this result has been stated with less than adequate precision. For example we should establish that simplifying the $k$ th convergent always leads to a fraction $p_{k} / q_{k}$ expressed in its lowest terms, and specify that this is the representation used in the proposition; also the introduction of $m$, not an integer, leads to a nonsimple continued fraction. Formally, the $p_{k}$ and $q_{k}$ are best considered as polynomials in the indeterminates $n_{0}, n_{1}, \ldots, n_{k}$ and the proof establishes that $p_{k+1}=$ $n_{k+1} p_{k}+p_{k-1}, q_{k+1}=n_{k+1} q_{k}+q_{k-1}$. An easy manipulation then shows that $p_{k+1} q_{k}-p_{k} q_{k+1}=(-1)^{k+1}$ and thus, inductively, that $p_{k+1}$ and $q_{k+1}$ are coprime, either as integers or as polynomials. In fact the polynomials $p_{k}$ and $q_{k}$ are irreducible. $^{108}$

108 A formal treatment can be found in many textbooks on number theory, for example, see Davenport (1968), pp. 77-113; Stark (1978), pp. 181-256 follows a geometrical approach close in spirit to ours. For the more advanced theory see Khinchin (1964). 


\section{A 4. Anthyphairesis.}

Algorithm 3. If $a$ and $b$ are two homogeneous magnitudes write $a-n_{0} b$ $=b_{1}$ with $b_{1}<b_{2}$. If $b_{1} \neq 0$, write $b-n_{1} b_{1}=b_{2}$ with $b_{2}<b_{1}$, etc. If at any stage $b_{k}=0$ the process terminates; this, it is very easy to see (and it is proved in $\mathrm{X}, 3$ ) corresponds to the situation where $a$ and $b$ are commensurable, and gives the standard procedure for determining their common measure; otherwise $a$ and $b$ are incommensurable $(\mathrm{X}, 2)$.

This process is very similar to Algorithm 2. In fact, if we assign real numbers to the two magnitudes so that the ratio $a: b$ is identified with the number $a / b=\theta$, and apply Algorithm 2 we get $a / b=n_{0}+\theta_{1}$ where $0 \leqslant \theta_{1}<1$, so $a-n_{0} b=b_{1}$, where $b_{1}=\theta_{1} b<b, 1 / \theta_{1}=n_{1}+\theta_{2}$ where $0 \leqslant$ $\theta_{2}<1$ so $b-n_{1} b_{1}=b_{2}$ where $b_{2}=\theta_{2} b_{1}<b_{1}$, etc. Hence the sequence of integers $\left[n_{0}, n_{1}, \ldots\right]$ thus generated by anthyphairesis is the sequence of denominators of the continued fraction expansion of $a / b$. Note however how anthyphairesis can be effected without having resource to representations of $a, b$, or $a / b$ as numbers in the case where, for example, $a$ and $b$ are two rectilinear magnitudes, using the theory of application of areas.

Write $\operatorname{Anth}(a, b)=\left[n_{0}, n_{1}, n_{2}, \ldots\right]$ to denote the anthyphairesis of $a$ and $b$.

A 5. Examples. Let us analyse the arithmetic behind examples of $\S 7$ in the light of these results.

(a) $\operatorname{Anth}(7921,4050)=[1,1,21,1,2,29,2]$. Aristarchus wants a lower bound for the ratio. Now the upper bound $[1,1]$ will be unusually good, since it precedes a long run of 21 steps; the next upper bound [1, 1, 21, 1] will be better, and the next step of Algorithm 1 will give a lower bound whose $a$ priori accuracy is the same (see Figure 4). Hence

$$
7921: 4050>[1,1,21,2]=88: 45 \text {. }
$$

The next case is more straightforward:

Anth $(71755875,61735500)=[1,6,6,4,1,2,1,2,1,6]$. (In fact, both numbers are divisible by 3375 , giving the ratio $21261: 18292)$. Truncating at $[1,6,6]$ will give an underestimate,

$$
71755875: 6173550>[1,6,6]=43: 37 \text {. }
$$

(b) The continued fraction expansion of $\sqrt{ } 3$ (see $\S A 2$ ) is $\sqrt{ } 3=$ $[1,1,2,1,2, \ldots]$, with convergents $\frac{1}{1}, \frac{2}{1}, \frac{5}{3}, \frac{7}{4}, \frac{19}{11}, \frac{26}{15}, \frac{71}{41}, \frac{97}{56}, \frac{265}{153}, \frac{362}{209}, \frac{989}{571}, \frac{1351}{780}$, $\frac{3691}{2131}, \ldots$ The ninth and twelf th will be under- and overestimates respectively.

A shorter and more plausible procedure is to use $3 \sqrt{ } 3: 1=\sqrt{ } 27: 1=$ $[5,5,10,5,10, \ldots]$, with convergents $\frac{5}{1}, \frac{26}{5}, \frac{265}{51}, \frac{1351}{260}, \ldots$ The third and fourth terms of this sequence will be under- and overestimates respectively; dividing the sequence by 3 gives every third term of the sequence of convergents of $\sqrt{ } 3: 1$ and, in particular, the two required rational approximations. $^{109}$

109 This was pointed out to me by D. T. Whiteside. The suggestive simple pattern of this example-dividing the convergents of $3 \sqrt{ } 3: 1$ by 3 gives every third convergent of $\sqrt{ } 3: 1$ -does not generalise to other cases, as evaluating the convergents of $\sqrt{ } 5: 1, \sqrt{ } 20: 1$ and $\sqrt{ } 45: 1$ will illustrate, though obvious heuristic considerations will lead to improved procedures for finding rational approximations to quadratic surds. 
(c) The procedure of 'bringing down to small numbers' referred to by Hero is almost certainly that used by Aristarchus in the first example above, and so would be equivalent to converting a truncated anthyphairesis into a quotient of two integers.

(d) The anthyphairesis of the diameter and side of a square was evaluated in $\$ 6$ and $\mathrm{A} 2$ :

$$
\text { Anth(diameter, side })=[1,2,2,2, \ldots] \text {, }
$$

and thus has convergents $\frac{1}{1}, \frac{3}{2}, \frac{7}{5}, \frac{17}{12}$, etc., the quotients of successive side and diameter numbers.

Now consider the error analysis of the side and diameter numbers described by Theon of Smyrna and Proclus. Theon emphasizes the result $d_{k}^{2}-2 s_{k}^{2}= \pm 1$, and Proclus observes that this can be established using II, 10. We can extend this further, by observing

$$
\frac{d_{k+1}}{s_{k+1}}-\frac{d_{k}}{s_{k}}=\frac{2 s_{k}^{2}-d_{k}^{2}}{s_{k} s_{k+1}}=\frac{ \pm 1}{s_{k} s_{k+1}},
$$

and this is precisely our Proposition 2, applied to this particular case. ${ }^{110}$

\section{REFERENCES}

1. Alexander of Aphrodisias, In topica, edited by M. Wallies, Berlin, 1891.

2. Aristotle, Topics, edited and translated by E. S. Forster, Loeb Classical Library Series, Heinemann, London and Harvard Univ. Press, Cambridge, Mass., 1960.

3. O. Becker, Eudoxos-Studien. I-IV, and other articles, Quellen und Studien zur Geschichte der Mathematik, Astronomie und Physik 2 (1933); 3 (1936).

4. W. Burkert, Lore and science in ancient Pythagoreanism, translated by E. L. Minar, Jr., Harvard Univ. Press, Cambridge, Mass., 1972.

5. H. S. M. Coxeter, Introduction to geometry, Wiley, New York, 1961.

6. H. Davenport, The higher arithmetic, Hutchinson, London, 1968.

7. H. Diels and W. Kranz, Die Fragmente der Vorsokratiker, 6th ed., Weidmannsche, Berlin, $1951-1952$.

8. Euclid, Elements, 3 vols., edited and translated by T. L. Heath, Cambridge Univ. Press, New York, 1926; reprint, Dover, New York, 1956.

9. T. Fletcher, Approximating by vectors (1), (2), Math. Teaching 63 (1973), 4-9; ibid., 64 (1973), 42-44.

10. D. H. Fowler, Book II of Euclid's Elements and a pre-Eudoxan theory of ratio (to appear).

11. K. von Fritz, The discovery of incommensurability by Hippasus of Metapontum, Ann. of Math. (2) 46 (1945), 242-264; reprinted in Studies in pre-Socratic philosophy, vol. 1, edited by D. J. Furley and R. E. Allen, Routledge and Kegan Paul, London, 1970, pp.382-412.

12. C. F. Gauss, Werke, 12 vol., Olms, Hildesheim, reprint, 1973.

13. R. W. Gosper, Continued fraction arithmetic, Item $101 \mathrm{~B}$ in $H A K M E M$, Artificial Intelligence Memo No. 239, A. I. Laboratory, M. I. T., Cambridge, Mass., February 29, 1972.

14. M. Hall, On the sum and product of continued fractions, Ann. of Math. (2) 48 (1947), 966-993.

15. T. L. Heath, A history of Greek mathematics, 2 vol., Oxford Univ. Press, London, 1921.

16. A. Hurwitz, Über die Kettenbruch-Entwicklung der Zahl e, Phys.-okon. Ges. Konigsberg, 1891; reprint, Mathematische Werke, Bd. 2, Birkhäuser, Basel, 1933, pp. 129-133.

110 Further instances of similar approximation procedures with their error analyses, together with a detailed and different analysis of Archimedes' approximations of $\sqrt{ } 3$, are given in Knorr (1976). 
17. A. Ya. Khinchin [= A. Ja. Hinčin], Continued fractions, Univ. of Chicago Press, Chicago, IIl., 1964.

18. F. Klein, Elementary mathematics from an advanced standpoint. vol. I, Arithmetic, algebra, analysis, 3rd ed.; reprint, Dover, New York, 1945.

19. J. Klein, Greek mathematical thought and the origin of algebra, translated by E. Brann, M. I. T. Press, Cambridge. Mass., 1968.

20. W. R. Knorr, The evolution of the Euclidean elements, Reidel, Dordrecht, 1975.

21. Archimedes and the measurement of the circle:A new interpretation, Arch. History Exact Sci. 15 (1976), 115-140.

22. __ Archimedes and the pre-Euclidean proportion theory, Transcript of a lecture to the Colloquium on the History of Mathematics at the Courant Institute, 1977.

23. D. E. Knuth, The art of computer programming, vol. 2, Seminumerical algorithms, AddisonWesley, Reading, Mass., 1969.

24. Pappus, The commentary of Pappus on Book X of Euclid's Elements, edited and translated by G. Junge and W. Thompson, Harvard Univ. Press, Cambridge, Mass., 1930; reprint, Johnson Reprint Corporation, New York and London, 1968.

25. Proclus, A commentary on the first book of Euclid's Elements, translated by G. N. Morrow, Princeton Univ. Press, Princeton, N. J., 1970.

26. Plato, Loeb Classical Library Series, Charmides (W. R. M. Lamb, 1927); Gorgias (W. R. M. Lamb, 1925); Laws (R. G. Bury, 2 vol., 1926); Lesser Hippias (H. N. Fowler, 1926); Philebus (H. N. Fowler, 1925); Protagoras (W. R. M. Lamb, 1924); Republic (P. Shorey, 2 vol., 1930-1935); Theaetetus (H. N. Fowler, 1921), Heinemann, London and Harvard Univ. Press., Cambridge, Mass.

27. E. B. Plooij, Euclid's conception of ratio and his definition of proportional magnitudes as criticized by Arabian commentators, Rotterdam, 1950.

28. G. N. Raney, On continued fractions and finite automata, Math. Ann. 206 (1973), 265-283.

29. H. J. S. Smith, Notes on continued fractions, Messenger of Mathematics, Ser. II, vol. 6, 1876, 1-14; reprint, Collected Works, vol. II, Chelsea, New York, 1965, pp. 135-147.

30. H. M. Stark, An introduction to number theory, Markham, Chicago, Ill, 1970; reprint M.I.T. Press, Cambridge, Mass., 1978.

31. A. E. Taylor, Forms and numbers: A study in Platonic metaphysics, Mind 35 (1926), 419-440; ibid., 36 (1927), $12-33$.

32. I. Thomas [ = I. Bulmer-Thomas], editor and translator, Selections illustrating the history of Greek mathematics, 2 vol., Loeb Classical Library Series, Heinemann, London and Harvard Univ. Press, Cambridge, Mass., 1939-1941.

33. D'A. W. Thompson, Excess and defect: Or the little more and the little less, Mind 38 (1929), $43-55$.

34. B. L. van der Waerden, Science awakening, translated by A. Dresden, Noordhoff, Groningen, 1954.

35. E. C. Zeeman, Research ancient and modern, Bull. Inst. Math. Appl. 10 (1974), 272-281; reprinted in Catastrophe Theory-Selected Papers 1972-1977, Addison-Wesley, Reading, Mass., 1977.

Mathematics InStitute, University OF WarWick, Coventry, ENGLAND 